\title{
Second Order Linear Difference Equations
}

\author{
A. M. Encinas and M. J. Jiménez \\ Departament de Matemàtiques, Universitat Politècnica de Catalunya
}

\begin{abstract}
ARTICLE HISTORY
Compiled October 11, 2017

ABSTRACT

We provide the explicit solution of a general second order linear difference equation via the computation of its associated Green function. This Green function is completely characterized and we obtain a closed expression for it using functions of two-variables, that we have called Chebyshev functions due to its intimate relation with the usual one-variable Chebyshev polynomials. In fact, we show that Chebyshev functions become Chebyshev polynomials if constant coefficients are considered.
\end{abstract}

\section{KEYWORDS}

Second order difference equations, Green function, initial value problem, Chebyshev functions, Chebyshev polynomials.

\section{Introduction}

Second order linear difference equations appear in mathematics and in sciences in both contexts, pure and applied. For instance, homogeneous equations with constant coefficients have generated an enormous amount of production in relation with several numbers sequences as, Horadam, Fibonacci, Lucas, Pell, Jacobsthal, to mention only a few, see [19]. Moreover, general homogeneous second order linear difference equations also appear related with combinatorial problems and encompasses the recurrences generating many famous combinatorial numbers, such as Fine, central Delannoy, Schröder, Motzkin, counting directed animals and derangements numbers, that play an important role in enumerative combinatorics and count several combinatorial objects, see for instance $[4,13]$ and references therein for the origin and the meaning of these sequences.

On the other hand, in the framework of applied mathematics, homogeneous second order linear equations are related with the so-called three term recurrences and hence with special functions, orthogonal polynomials and Jacobi matrices that play an important role in the mathematical models of systems that appear in various branches of engineering. Even equations with constant coefficients are closely related with the ubiquitous Chebyshev polynomials that are present in many areas of applied science. Moreover, second order linear difference equations correspond to the finite difference discretization for the general second order linear differential equation. So, they are widely used for the numerical analysis and approximation of the corresponding physical application.

Closed form solutions of homogeneous second order equations with constant coefficients are well known and widely used. Many papers are devoted to this specific topic, where the so called Binet Formula, and hence the roots of the characteristic polynomial, appears as the starting point, see for instance [19]. In this case, we can apply that any homogeneous second order equation with constant coefficients is equivalent to 
a Chebyshev recurrence to obtain all solutions as suitable combinations of Chebyshev polynomials, see [2], although other approaches, based on the equivalence with a first order system, are also avaible, see [11]

In case of equations with variable coefficients, the closed form solution of the first order equation is well-known and easy to obtain. For second order equations the only easy case appears when a non trivial solution is known, since then the standard reduction of the orden technique, allows us to obtain another linearly independent solution. However, in the general case closed formulas are more scarce and usually very difficult to treat with. For instance, in [14], R.J. Mallik developed a formula expressing the solutions solely in terms of the coefficients of the equation. His methodology has an strong combinatorial flavor and it is based in the construction of suitable uples of integers to used them as set of indexes. Independently, I. Godoskov gave in [9] two approaches based first in a canonical reduction of the given equation into another one with only a coefficient and then either on recursive sums or on which the author calls discrete dimensional-convolution procedure. In the very special case when the variable coefficients are periodic, the authors have recently proved that the given equation is essentially equivalent to another one with constant coefficients, specifically to a Chebyshev equation which parameter can be computed through a sum-formula depending only on the coefficients, see [7]. As a by-product the characterization of the existence of periodic solutions was obtained.

All the above mentioned works treat only with homogeneous equations, although in [15] R.J. Mallik also considered the non-homogeneous case.

In this work we obtain a new closed formula for the solution of general second order linear difference equations. First we follow the guidelines of the differential case and hence all ours results gravitate around the Green function for the equation. Although this concept was already implicit in [5], to the best of our knowledge this is the first time in which the definition of the Green function is established. Since the Green function allows us to obtain a solution for the non-homogeneous equation and all the solutions for the homogeneous one, our main aim is to obtain a closed formula for the computation of the Green function. Besides, we also describe many additional properties. In particular, we show how the knowledge of the Green function completely determines the coefficients of the equation and moreover we characterize those functions that are the Green function of some equation.

Our technique is mainly combinatorial and hence it is closer to Mallik's than to Gonoskov's, and we strongly believe that the obtained expression is more clear and simple than Mallik's one. We adapt the concept of binary multi-index introduced in [7] to obtain what we have called Chebyshev functions through which the Green functions are expressed. When the obtained formula is applied to the case of constant coefficients, it becomes the classical (a single variable) Chebyshev polynomials. In this particular case, we obtain some result that seem be new, as the characterization of this kind of equations as shift-invariant.

We have developed our results for equations with coefficients in a field of characteristic different of 2. Due to the combinatorial nature of the techniques, many of them are also valid for equations with coefficients in an integral domain or even in more general rings, but to do this probably we would need strong constrains on the coefficients.

\section{Preliminaries}

Throughout the paper, $(\mathbb{K},+, \cdot)$ denotes a (commutative) field with characteristic not equal to 2 . As usual, the additive and the multiplicative identities of $\mathbb{K}$ are denoted by 0 and 1 , respectively. If $a \in \mathbb{K}^{*}=\mathbb{K} \backslash\{0\}$ its inverse is denoted by $a^{-1}$ and also by $\frac{1}{a}$. Moreover, $\mathbb{N}$ denotes the set of non-negative integers and $\mathbb{N}^{*}=\mathbb{N} \backslash\{0\}$. We always assume the usual conventions $0^{0}=1$ and that empty sums and empty products are defined as 0 and 1 , respectively. Therefore, $\sum_{k=i}^{j} a_{k}=0$ and $\prod_{k=i}^{j} a_{j}=1$ when $j<i$.

A sequence of elements of $\mathbb{K}$ is a function $a: \mathbb{N} \longrightarrow \mathbb{K}$. We denote by $\ell(\mathbb{K})$ the space of all sequences of elements of $\mathbb{K}$ and by $\ell\left(\mathbb{K}^{*}\right) \subset \ell(\mathbb{K})$ the subset of the sequences $a \in \ell(\mathbb{K})$ such that $a(k) \neq 0$ for all $k \in \mathbb{N}$. 
Clearly $(\ell(\mathbb{K}),+, \cdot)$ is a ring with identity with the element-wise sum and product of sequences. Therefore, the additive identity in $\ell(\mathbb{K})$ is the null sequence, whereas the multiplicative identity of $\ell(\mathbb{K})$ is the constant sequence whose entries are all equals 1 . Moreover, identifying $a \in \mathbb{K}$ with the constant sequence whose value at any $k \in \mathbb{N}$ is $a$, we get that $\mathbb{K} \subset \ell(\mathbb{K})$ is a subring of $\ell(\mathbb{K})$. Through the paper, we use this identification and hence if $a \in \mathbb{K}$, we denote the corresponding constant sequence also by $a$. With this convention in mind, 0 is the additive identity in $\ell(\mathbb{K})$, whereas the multiplicative identity of $\ell(\mathbb{K})$ is 1 . Clearly, $\ell\left(\mathbb{K}^{*}\right)$ is the group of units of $\ell(\mathbb{K})$ and moreover if $a \in \ell\left(\mathbb{K}^{*}\right)$, then its multiplicative inverse is denoted by $a^{-1}$.

Given $a \in \ell(\mathbb{K})$ and $m \in \mathbb{N}$, the sequence $a_{m}$ defined as $a_{m}(k)=a(k+m)$ for any $k \in \mathbb{N}$ is called the $m$-shift of $a$. Clearly, $a_{0}=a, a_{r+s}=\left(a_{r}\right)_{s}$ for any $r, s \in \mathbb{N}$ and $a \in \ell(\mathbb{K})$ is constant iff $a=a_{1}$. On the other hand, when $a \in \ell\left(\mathbb{K}^{*}\right)$, then $a_{m} \in \ell\left(\mathbb{K}^{*}\right),\left(a_{m}\right)^{-1}=\left(a^{-1}\right)_{m}$ and this sequence is denoted by $a_{m}^{-1}$.

For any sequence $a \in \ell(\mathbb{K})$ and any $k, m \in \mathbb{N}$, we define the definite integral of a between $k$ and $m$ as the value

$$
\int_{m}^{k} a(s) \nabla s=\operatorname{sign}(k-m) \sum_{s=\min \{k, m\}+1}^{\max \{k, m\}} a(s)
$$

where $\operatorname{sign}(0)=0, \operatorname{sign}(-k)=-1$ and $\operatorname{sign}(k)=1$ for any $k \in \mathbb{N}^{*}$, see [18] for a slightly different definition of the definite integral of a sequence. In particular, for any $k \in \mathbb{N}$ we have that

$$
\int_{k}^{k} a(s) \nabla s=0, \quad \int_{k}^{k+1} a(s) \nabla s=a(k+1) \quad \text { and } \quad \int_{k}^{k-1} a(s) \nabla s=-a(k) .
$$

Moreover, for any $k, m, r \in \mathbb{N}$ we have that

$$
\int_{m}^{k} a(s) \nabla s+\int_{k}^{r} a(s) \nabla s=\int_{m}^{r} u(s) \nabla s
$$

which taken $r=m$, implies that $\int_{k}^{m} a(s) \nabla s=-\int_{m}^{k} a(s) \nabla s$ for any $k, m \in \mathbb{N}$.

The definite integral is a linear and monotone functional, since for any $a \in \ell(\mathbb{K})$

$$
\left|\int_{k}^{m} a(s) \nabla s\right| \leq\left|\int_{k}^{m}\right| a(s)|\nabla s|, \text { for any } k, m \in \mathbb{N} .
$$

Given $a \in \ell(\mathbb{K})$ if we define $A \in \ell(\mathbb{K})$ as $A(k)=\int_{0}^{k} a(s) \nabla s$, then $A(k+1)-A(k)=a(k+1)$, for any $k \in \mathbb{N}$. Conversely, we have the following discrete version of the Fundamental Theorem of Calculus: Given $f \in \ell(\mathbb{K})$ then $x \in \ell(\mathbb{K})$ satisfies that $x(k)-x(k-1)=f(k)$ for any $k \in \mathbb{N}^{*}$ iff there exists $\alpha \in \mathbb{K}$ such that

$$
x(k)=\alpha+\int_{0}^{k} f(s) \nabla s, \text { for any } k \in \mathbb{N}
$$

In particular, given $\beta \in \mathbb{K}$, then $x \in \ell(\mathbb{K})$ satisfies that $x(k)-x(k-1)=\beta$ for any $k \in \mathbb{N}^{*}$ iff there exists $\alpha \in \mathbb{K}$ such that $x(k)=\alpha+\beta k$ for any $k \in \mathbb{N}$. 


\section{Linear first order difference equations}

Although the main objective of this work is to provide closed formulas for the solution of arbitrary linear second order difference equations, for the sake of completeness we start considering the case of linear first order difference equations. Indeed, the fundamental theorem of calculus is the most simple case of this kind of problems. In spite of being a case simpler than the second order one, already appear both the necessity of introducing some hypotheses on the coefficients and concepts that will be relevant in the analysis of second order equations.

Given $a, c, f \in \ell(\mathbb{K})$ the first order linear difference equation with coefficients a, $c$ and data $f$ consists in finding all sequences $x \in \ell(\mathbb{K})$ satisfying the identity

$$
a(k) x(k)=c(k-1) x(k-1)+f(k), \quad k \in \mathbb{N}^{*} .
$$

Each sequence $x \in \ell(\mathbb{K})$ satisfying the above identity is called a solution of the difference equation. Note that any solution of the Equation (1) does not depend on the values $a(0)$ and $f(0)$.

One of the main problems related with Equation (1) is the so called initial value problems that refers to determine a solution from a given value. Specifically, the initial value problem at $m \in \mathbb{N}$ ask for a solution of (1) satisfying that $x(m)=\alpha$ for a given $\alpha \in \mathbb{K}$. Clearly, to determine $x$ from (1) and $\alpha$ we must to impose conditions on the coefficients $a$ and $c$. So, fixed $m$ to obtain $x(k)$ for $k>m$ is necessary that $a(k) \neq 0$, whereas to obtain $x(k)$ for $k<m$ we need that $c(k-1) \neq 0$. Moreover, since we wish to solve any initial value problem it is reasonable to demand that $a, c \in \ell\left(\mathbb{K}^{*}\right)$. Under this hypothesis it is easy to check that

$$
x(k)= \begin{cases}\alpha \prod_{j=m}^{k-1} \frac{c(j)}{a(j+1)}+\sum_{s=m+1}^{k} \frac{f(s)}{a(s)} \prod_{j=s}^{k-1} \frac{c(j)}{a(j+1)}, \quad k \geq m, \\ \alpha \prod_{j=k}^{m-1} \frac{a(j+1)}{c(j)}-\sum_{s=k+1}^{m} \frac{f(s)}{a(s)} \prod_{j=k}^{s-1} \frac{a(j+1)}{c(j)}, \quad k \leq m,\end{cases}
$$

which in particular implies that for any $f \in \ell(\mathbb{K})$, any $m \in \mathbb{N}$ and any $\alpha \in \mathbb{K}$ the corresponding initial value problem has a unique solution.

The above expression motivates us to consider the function $g: \mathbb{N} \times \mathbb{N} \longrightarrow \mathbb{K}^{*}$ defined as

$$
g(k, s)=\frac{1}{a(s)}\left(\prod_{j=\min \{k, s\}}^{\max \{k, s\}-1} \frac{c(j)}{a(j+1)}\right)^{\operatorname{sign}(k-s)}
$$

and that we name Green function of the initial value problem for Equation (1). Notice that fixed $s \in \mathbb{N}$, the sequence $x(k)=g(k, s)$ is the unique solution of the Equation (1) satisfying $x(s)=a(s)^{-1}$. Clearly $g$ depends on $a$ and $c$ and hence it should be denoted as $g_{a, c}$. Since we assume the coefficients $a$ and $c$ are fixed, for the sake of simplicity we have dropped the subindexes $a, c$.

With the above notations and hypotheses we have the following key result about initial value problems for first order linear difference equations.

Proposition 3.1. Given $a, c \in \ell\left(\mathbb{K}^{*}\right)$, then for any $\alpha \in \mathbb{K}$, any $f \in \ell(\mathbb{K})$ and any $m \in \mathbb{N}$, the unique solution of the initial value problem

$$
a(k) x(k)=c(k-1) x(k-1)+f(k), \quad k \in \mathbb{N}^{*}, \quad x(m)=\alpha
$$


is given by

$$
x(k)=\alpha \frac{g(k, m)}{g(m, m)}+\int_{m}^{k} g(k, s) f(s) \nabla s, \quad k \in \mathbb{N} .
$$

Taking into account that when the coefficients are constant, then

$$
g(k, s)=\frac{1}{a}\left(\frac{c}{a}\right)^{k-s}, \quad k, s \in \mathbb{N},
$$

and we have the following particular case.

Corollary 3.2. Given $a, c \in \mathbb{R}^{*}$, then for any $\alpha \in \mathbb{K}$, any $f \in \ell(\mathbb{K})$ and any $m \in \mathbb{N}$, the unique solution of the initial value problem

$$
a x(k)-c x(k-1)=f(k), \quad k \in \mathbb{N}^{*}, \quad x(m)=\alpha
$$

is given by

$$
x(k)=\alpha\left(\frac{c}{a}\right)^{k-m}+\frac{1}{a} \int_{m}^{k}\left(\frac{c}{a}\right)^{k-s} f(s) \nabla s=\left(\frac{c}{a}\right)^{k}\left[\alpha\left(\frac{a}{c}\right)^{m}+\frac{1}{a} \int_{m}^{k}\left(\frac{a}{c}\right)^{s} f(s) \nabla s\right], \quad k \in \mathbb{N} .
$$

Notice that when $a=c=1$, then $g(k, s)=1$ for any $k, s \in \mathbb{N}$ and moreover, the above identity coincides with the fundamental theorem of calculus.

\section{Initial value problems and Green function for second order equations}

Given three sequences $a, c \in \ell\left(\mathbb{K}^{*}\right)$ and $b \in \ell(\mathbb{K})$, for any $f \in \ell(\mathbb{K})$, the second order difference equation with coefficients $a, b, c$ and data $f$ consists in finding all sequences $x \in \ell(\mathbb{K})$ satisfying the identity

$$
a(k) x(k+1)-b(k) x(k)+c(k-1) x(k-1)=f(k), \quad k \in \mathbb{N}^{*} .
$$

Any sequence $x \in \ell(\mathbb{K})$ satisfying the above identity is called a solution of the difference equation. Note that any solution of the Equation (2) does not depend on the value of the coefficients $a, b$ and the data $f$ at $k=0$.

We say that Equation (2) has constant coefficients when $a, c \in \mathbb{K}^{*}$ and $b \in \mathbb{K}$.

The equation

$$
a(k) x(k+1)-b(k) x(k)+c(k-1) x(k-1)=0, \quad k \in \mathbb{N}^{*},
$$

corresponds to take as data the null function in Equation (2), is called homogeneous and we refer it as the homogeneous equation associate with (2). Clearly the null sequence is always a solution of the homogeneous equation and we refer it as the trivial solution.

Throughout this section we consider fixed the coefficients $a, c \in \ell\left(\mathbb{K}^{*}\right)$ and $b \in \ell(\mathbb{K})$. The first result establishes that each solution of any equation with coefficients $a, b$ and $c$ is completely determined by its values at two consecutive indexes.

Lemma 4.1. Given $m \in \mathbb{N}$ and $\alpha, \beta \in \mathbb{K}$, for any $f \in \ell(\mathbb{K})$ the second order difference equation

$$
a(k) x(k+1)-b(k) x(k)+c(k-1) x(k-1)=f(k), \quad k \in \mathbb{N}^{*}
$$


has a unique solution $x \in \ell(\mathbb{K})$ such that $x(m)=\alpha, x(m+1)=\beta$. In particular, when $f=0$, then $x=0$ iff there exists $m \in \mathbb{N}$ such that $x(m)=x(m+1)=0$.

Given $m \in \mathbb{N}, \alpha, \beta \in \mathbb{K}$, for any $f \in \ell(\mathbb{K})$, the problem of obtaining the solutions of Equation (2) satisfying that $x(m)=\alpha$ and $x(m+1)=\beta$ is known as the initial value problem for the Equation (2) at $m$ with data $f$ and initial data $\alpha$ and $\beta$. Therefore, the above lemma says that any initial value problem for the Equation (2) has a unique solution. Clearly, if $x \in \ell(\mathbb{K})$ is solution of Equation (2) and we consider the values $\alpha=x(m)$ and $\beta=x(m+1)$, where $m \in \mathbb{N}$, then $x$ is the unique solution of this initial value problem. So, determining the solution of any initial value problem at any $m \in \mathbb{N}$ is equivalent to obtaining all the solutions of the Equation (2).

In the sequel we denote by $\mathcal{S}$ the set of solutions of the homogeneous equation associated with (2); that is, $z \in \mathcal{S}$ iff

$$
a(k) z(k+1)-b(k) z(k)+c(k-1) z(k-1)=0, \quad k \in \mathbb{N}^{*} .
$$

Moreover, for any $f \in \ell(\mathbb{K})$ we denote by $\mathcal{S}(f)$ the set of solutions of the Equation (2) with data $f$. We remark that $\mathcal{S}$ and $\mathcal{S}(f)$ depend on $a, b$ and $c$ and hence it should be denoted as $\mathcal{S}_{a, b, c}$ and $\mathcal{S}_{a, b, c}(f)$, respectively. Since we assume the coefficients of the Equation (2) are fixed, for the sake of simplicity we drop the subindexes $a, b$ and $c$ in the above notations. The following result reflects the linear character of the Equation (2).

Proposition 4.2 (Superposition Principle). The set $\mathcal{S}$ is a two dimensional $\mathbb{K}$-vector space and for any $f \in \ell(\mathbb{K})$ then we get $\mathcal{S}(f)=x+\mathcal{S}$, where $x \in \mathcal{S}(f)$. Moreover, given $m \in \mathbb{N}$ and $\alpha, \beta \in \mathbb{K}$, if $x \in \ell(\mathbb{K})$ is the unique solution of the initial value problem

$$
a(k) x(k+1)-b(k) x(k)+c(k-1) x(k-1)=f(k) ; \quad k \in \mathbb{N}^{*}, \quad x(m)=\alpha, x(m+1)=\beta,
$$

then $x=y+z$ where $z$ is the unique solution of the initial value problem for the homogeneous equation

$$
a(k) z(k+1)-b(k) z(k)+c(k-1) z(k-1)=0 ; \quad k \in \mathbb{N}^{*}, \quad z(m)=\alpha, \quad z(m+1)=\beta,
$$

and $y$ is the unique solution of the initial value problem

$$
a(k) y(k+1)-b(k) y(k)-c(k-1) y(k-1)=f(k) ; \quad k \in \mathbb{N}^{*}, \quad y(m)=y(m+1)=0 .
$$

Next we introduce the mail tool for the resolution of any initial value problem. We call the Green function for the difference Equation (3), or simply the Green function, the function $g: \mathbb{N} \times \mathbb{N} \longrightarrow \mathbb{K}$ defined for any $s \in \mathbb{N}$ as $g(\cdot, s)$, the unique solution of the homogeneous Equation (3) satisfying $g(s, s)=0$ and $g(s+1, s)=a(s)^{-1}$. Notice that for any $s \in \mathbb{N}$, from the identity

$$
a(s+1) g(s+2, s+1)-b(s+1) g(s+1, s+1)+c(s) g(s, s+1)=0,
$$

we obtain that $g(s, s+1)=-c(s)^{-1}$, whereas from the identity

$$
a(s+1) g(s+2, s)-b(s+1) g(s+1, s)+c(s) g(s, s)=0,
$$

we obtain that $b(s+1)=a(s+1) a(s) g(s+2, s)$. Therefore, the coefficients of the equation determine its Green function and conversely, the Green function completely determines the coefficients of the equation, 
since the value $b(0)$ is not relevant to solve the equation. Explicitly, we have that

$$
a(k)=\frac{1}{g(k+1, k)}, \quad c(k)=\frac{-1}{g(k, k+1)}, \quad k \in \mathbb{N} \quad \text { and } \quad b(k)=\frac{g(k+1, k-1)}{g(k+1, k) g(k, k-1)}, \quad k \in \mathbb{N}^{*} .
$$

We remark that since the Green function depends on $a, b$ and $c$ it should be denoted as $g_{a, b, c}$. Again we have dropped the subindexes $a, b$ and $c$ for the sake of simplicity.

The relevance of the Green function is showed in the next result; compare it with the corresponding to initial value problems for first order difference equations.

Theorem 4.3. Given $\alpha, \beta \in \mathbb{K}$ and $f \in \ell(\mathbb{K})$, the unique solution of the initial value problem

$$
a(k) x(k+1)-b(k) x(k)+c(k-1) x(k-1)=f(k) ; \quad k \in \mathbb{N}^{*}, \quad x(m)=\alpha, x(m+1)=\beta,
$$

is given by

$$
x(k)=\alpha \frac{g(k, m+1)}{g(m, m+1)}+\beta \frac{g(k, m)}{g(m+1, m)}+\int_{m}^{k} g(k, s) f(s) \nabla s, \quad k \in \mathbb{N} .
$$

Proof. If we consider the sequence $z(k)=\beta a(m) g(k, m)-\alpha c(m) g(k, m+1), k \in \mathbb{N}$, it is clear that $z \in \mathcal{S}$ and moreover $z(m)=\alpha$ and $z(m+1)=\beta$. Therefore, from the Superposition Principle, it is enough to prove that $y \in \ell(\mathbb{K})$ defined as $y(k)=\int_{m}^{k} g(k, s) f(s) \nabla s$ for any $k \in \mathbb{N}$, satisfies that $y \in \mathcal{S}(f)$ and $y(m)=y(m+1)=0$.

Clearly $y(m)=0$ and moreover $y(m+1)=g(m+1, m+1) f(m+1)=0$. On the other hand, if given $k \in \mathbb{N}^{*}$ we consider the value $A(k)=a(k) y(k+1)-b(k) y(k)+c(k-1) y(k-1)$, then

$$
\begin{aligned}
A(k) & =a(k) \int_{m}^{k+1} g(k+1, s) f(s) \nabla s-b(k) \int_{m}^{k} g(k, s) f(s) \nabla s-c(k-1) \int_{m}^{k-1} g(k-1, s) f(s) \nabla s \\
& =\int_{m}^{k-1}[a(k) g(k+1, s)-b(k) g(k, s)-c(k-1) g(k-1, s)] f(s) \nabla s \\
& +\int_{k-1}^{k}[a(k) g(k+1, s)-b(k) g(k, s)] f(s) \nabla s+a(k) \int_{k}^{k+1} g(k+1, s) f(s) \nabla s \\
& =[a(k) g(k+1, k)-b(k) g(k, k)] f(k)+a(k) g(k+1, k+1) f(k+1)=f(k),
\end{aligned}
$$

since $a(k) g(k+1, s)-b(k) g(k, s)-c(k-1) g(k-1, s)=0$ for any $s \in \mathbb{N}$.

As an example, we consider the following initial value problem for an uncoupled equation, see Corollary 7.6 for the general case,

$$
(k+2) z(k+1)+k z(k-1)=f(k), \quad x(0)=\alpha, x(1)=\beta .
$$


The Green function for this uncoupled equations is

$$
\begin{aligned}
g(2 k+1,2 s) & =\frac{(-1)^{|k-s|}}{2 s+2}\left[\frac{\min \{k, s\}+1}{\max \{k, s\}+1}\right]^{\operatorname{sign}(k-s)} & , & k, s \in \mathbb{N}, \\
g(2 k, 2 s+1) & =\frac{(-1)^{|k-s|-1}}{2 k+1}, & & k, s \in \mathbb{N} \\
g(m, n) & =0, & & \text { otherwise. }
\end{aligned}
$$

and hence the unique solution of the above initial value problem is determined by

$$
\begin{aligned}
& x(2 k)=\frac{(-1)^{k}}{2 k+1}\left[\alpha+\sum_{s=0}^{k-1}(-1)^{s+1} f(2 s+1)\right], \quad k \in \mathbb{N}, \\
& x(2 k+1)=\frac{(-1)^{k}}{2(k+1)}\left[2 \beta+\sum_{s=1}^{k}(-1)^{s} f(2 s)\right], \quad k \in \mathbb{N} .
\end{aligned}
$$

We end this section by introducing a very useful concept for the analysis of linear second order difference equations. The Wronskian, also called the Casoratian see [1], is the map

$$
\begin{aligned}
\omega: \ell(\mathbb{K}) \times \ell(\mathbb{K}) & \longrightarrow \ell(\mathbb{K}) \\
(z, w) & \longrightarrow z w_{1}-z_{1} w ;
\end{aligned}
$$

that is,

$$
\omega[z, w](k)=z(k) w(k+1)-z(k+1) w(k), \quad \text { for any } k \in \mathbb{N} .
$$

It is clear that the wronskian is a bilinear skew-symmetric map. In addition, for any $u, w, z \in \ell(\mathbb{K})$ it is satisfied that $\omega[u z, u w]=u u_{1} \omega[z, w]$; that is

$$
\omega[u z, u w](k)=u(k) u(k+1) \omega[z, w](k), \quad \text { for any } k \in \mathbb{N} .
$$

Moreover, $\omega[z, w]=0$ when $z$ and $w$ are linearly dependent. The relation between the wronskian and the solution of the homogeneous difference Equation (3) is clarify in the sequel well-known result.

Proposition 4.4. If $z, w \in \mathcal{S}$, then it is satisfied that

$$
a(k) \omega[z, w](k)=c(0) \omega[z, w](0) \prod_{j=1}^{k-1} \frac{c(j)}{a(j)}, \quad k \in \mathbb{N}^{*} .
$$

In particular, $\omega[z, w] \in \ell\left(\mathbb{K}^{*}\right)$ iff $\omega[z, w](0) \in \mathbb{K}^{*}$ and this property is equivalent to be $\{z, w\}$ a basis of $\mathcal{S}$.

Proof. For any $k \in \mathbb{N}^{*}$ we have that

$$
\begin{aligned}
a(k) \omega[z, w](k) & =z(k)[b(k) w(k)-c(k-1) w(k-1)]-[b(k) z(k)-c(k-1) z(k-1)] w(k) \\
& =c(k-1)[z(k-1) w(k)-z(k) w(k-1)]=c(k-1) \omega[z, w](k-1),
\end{aligned}
$$


and hence $\omega[z, w]$ is a solution of the first order difference equation with coefficients $a$ and $c$. Therefore from Proposition 3.1, for any $k \in \mathbb{N}$ we have that

$$
\omega[z, w](k)=\omega[z, w](0) \prod_{j=0}^{k-1} a(j+1)^{-1} c(j)=a(k)^{-1} c(0) \omega[z, w](0) \prod_{j=1}^{k-1} a(j)^{-1} c(j)
$$

and hence, the invertibility property of the wronskian is then consequence of being $a, c \in \ell\left(\mathbb{K}^{*}\right)$.

Finally, from Theorem 4.3 we have that

$$
(z(k), w(k))=(g(k, 0), g(k, 1))\left[\begin{array}{cc}
0 & a(0) \\
-c(0) & 0
\end{array}\right]\left[\begin{array}{cc}
z(0) & w(0) \\
z(1) & w(1)
\end{array}\right]
$$

and when $\omega[z, w](0) \in \mathbb{K}^{*}$ we also have

$$
(g(k, 0), g(k, 1))=\omega[z, w](0)^{-1}(z(k), w(k))\left[\begin{array}{cc}
w(1) & -w(0) \\
-z(1) & z(0)
\end{array}\right]\left[\begin{array}{cc}
0 & -c(0)^{-1} \\
a(0)^{-1} & 0
\end{array}\right] .
$$

Therefore, $\omega[z, w](0) \in \mathbb{K}^{*}$ iff $\{z, w\}$ is a basis of $\mathcal{S}$.

Notice that the identity of the above Proposition also implies that $\omega[z, w] \in \ell\left(\mathbb{K}^{*}\right)$ iff $\omega[z, w](m) \in \mathbb{K}^{*}$ for some $m \in \mathbb{N}$.

Corollary 4.5. Given $s \in \mathbb{N}$, then $\{g(\cdot, s), g(\cdot, s+1)\}$ is a basis of $\mathcal{S}$. More generally, given $s, \hat{s} \in \mathbb{N}$, $\{g(\cdot, s), g(\cdot, \hat{s})\}$ is a basis of $S$ iff $g(\hat{s}, s) \neq 0$ or equivalently iff $g(s, \hat{s}) \neq 0$.

Proof. If $z=g(\cdot, s)$ and $w=g(\cdot, \hat{s})$, then

$$
\omega[z, w](\hat{s})=a(\hat{s})^{-1} g(\hat{s}, s) \quad \text { and } \quad \omega[z, w](s)=-a(s)^{-1} g(s, \hat{s})
$$

and the result is a direct consequence of the above Proposition.

Corollary 4.6. Consider $z, w \in \mathcal{S}$ a basis of $\mathcal{S}$. Then

$$
g(k, s)=a(s)^{-1} \omega[z, w](s)^{-1}[w(k) z(s)-z(k) w(s)], \text { for any } k, s \in \mathbb{N} .
$$

Proof. From Proposition 4.4 we know that $\omega[z, w] \in \ell\left(\mathbb{K}^{*}\right)$. On the other hand, fixed $s \in \mathbb{N}$ if we consider $u \in \ell(\mathbb{K})$ defined as $u=w z(s)-z w(s)$, then $u \in \mathcal{S}$. Since $u(s)=0$ and $u(s+1)=\omega[z, w](s)$, we finally conclude that $g(\cdot, s)=\omega[z, w](s)^{-1} a(s)^{-1} u$.

\section{Equivalent equations}

In the previous section we have shown that the knowledge of a pair of linearly independent solutions of a given linear homogeneous equation of second order completely determines the unique solutions of any initial value problems for arbitrary data. The objective of this section is to determine when two homogeneous difference equation of second order have the same solutions. In fact, we analyze a more general scenario in which we can obtain the solution of a given equation from the solution of another one, multiplying by a fixed sequence in $\ell\left(\mathbb{K}^{*}\right)$. 
Through this section we consider fixed the sequences $a, \hat{a}, c, \hat{c} \in \ell\left(\mathbb{K}^{*}\right)$ and $b, \hat{b} \in \ell(\mathbb{K})$, the homogeneous Equation (3) and

$$
\hat{a}(k) x(k+1)-\hat{b}(k) x(k)+\hat{c}(k-1) x(k-1)=0, \quad k \in \mathbb{N}^{*},
$$

and we denote by $\mathcal{S}$ and by $\widehat{\mathcal{S}}$ the spaces of solutions of the Equations (3) and (5), respectively.

Given $\nu \in \ell\left(\mathbb{K}^{*}\right)$ Equation (5) is called $\nu$-equivalent to (3) iff $\widehat{\mathcal{S}}=\nu \mathcal{S}$. Notice that the above identity says that $\{z, w\}$ is basis of $\mathcal{S}$ iff $\{\nu z, \nu w\}$ is basis of $\widehat{\mathcal{S}}$.

Clearly Equation (5) is $\nu$-equivalent to (3) iff Equation (3) is $\nu^{-1}$-equivalent to (5). So, when $\nu=1$ we drop the reference to the function and then we simply call the Equations (3) and (5) equivalents. Therefore, two homogeneous equations are equivalents iff they have the same solutions.

Clearly, if for any $\gamma \in \ell\left(\mathbb{K}^{*}\right)$ we define $\hat{a}=\gamma a, \hat{b}=\gamma b$ and $\hat{c}=\gamma_{1} c$, then the Equations (3) and (5) are equivalents (below we prove that the converse is true). In particular, choosing $\gamma=a^{-1}$ we obtain that any homogeneous second order difference equation is equivalent to other one in which the first coefficient is equal to 1 . We call explicit this kind of equations.

Since the main objective of this section is to study under which conditions the Equations (3) and (5) are $\nu$-equivalents, we first prove that the coefficients of any explicit homogeneous equation are determined by its solutions, or equivalently by any basis of the space of its solutions.

Proposition 5.1. Consider $z, w \in \ell(\mathbb{K})$ such that $\omega[z, w] \in \ell\left(\mathbb{K}^{*}\right)$. Given $c \in \ell\left(\mathbb{K}^{*}\right)$ and $b \in \ell(\mathbb{K})$, then $z$ and $w$ are solutions of the equation

$$
x(k+1)-b(k) x(k)+c(k-1) x(k-1)=0, \quad k \in \mathbb{N}^{*}
$$

iff it is satisfied that

$$
b(k)=\frac{w(k+1) z(k-1)-z(k+1) w(k-1)}{\omega[z, w](k-1)}, \quad k \in \mathbb{N}^{*} \quad \text { and } \quad c(k)=\frac{\omega[z, w](k+1)}{\omega[z, w](k)}, \quad k \in \mathbb{N} .
$$

Proof. Clearly $z$ and $w$ are solutions of the given equation iff

$$
\left[\begin{array}{c}
z(k+1) \\
w(k+1)
\end{array}\right]=\left[\begin{array}{cc}
z(k) & -z(k-1) \\
w(k) & -w(k-1)
\end{array}\right]\left[\begin{array}{c}
b(k) \\
c(k-1)
\end{array}\right], \quad \text { for any } k \in \mathbb{N}^{*}
$$

and hence iff

$$
\left[\begin{array}{c}
b(k) \\
c(k-1)
\end{array}\right]=\frac{1}{\omega[z, w](k-1)}\left[\begin{array}{cc}
-w(k-1) & z(k-1) \\
-w(k) & z(k)
\end{array}\right]\left[\begin{array}{c}
z(k+1) \\
w(k+1)
\end{array}\right], \quad \text { for any } k \in \mathbb{N}^{*}
$$

and the result follows.

Theorem 5.2. Given $\nu \in \ell\left(\mathbb{K}^{*}\right)$, the Equation (5) is $\nu$-equivalent to Equation (3) iff there exists $\gamma \in \ell\left(\mathbb{K}^{*}\right)$ such that

$$
\hat{a}=\gamma a, \quad \hat{b}=\gamma \nu_{1} \nu^{-1} b, \quad \hat{c}=\gamma_{1} \nu_{2} \nu^{-1} c .
$$

In particular, the Equation (5) and (3) are equivalents iff there exists $\gamma \in \ell\left(\mathbb{K}^{*}\right)$ such that $\hat{a}=\gamma a, \hat{b}=\gamma b$ and $\hat{c}=\gamma_{1} c$. 
Proof. Let $\{z, w\}$ a basis of $\mathcal{S}$. Since $\{\nu z, \nu w\}$ is a basis of $\widehat{\mathcal{S}}$, applying the above Proposition we get that

$$
\begin{aligned}
\frac{\hat{b}(k)}{\hat{a}(k)} & =\frac{\nu(k+1) \nu(k-1)[w(k+1) z(k-1)-z(k+1) w(k-1)]}{\nu(k) \nu(k-1) \omega[z, w](k-1)}=\frac{\nu(k+1)}{\nu(k)} \frac{b(k)}{a(k)}, \\
\frac{\hat{c}(k-1)}{\hat{a}(k)} & =\frac{\nu(k+1) \nu(k) \omega[z, w](k)}{\nu(k) \nu(k-1) \omega[z, w](k-1)}=\frac{\nu(k+1) \omega[z, w](k)}{\nu(k-1) \omega[z, w](k-1)}=\frac{\nu(k+1)}{\nu(k-1)} \frac{c(k-1)}{a(k)},
\end{aligned}
$$

for any $k \in \mathbb{N}^{*}$. The result follows taking $\gamma=a^{-1} \hat{a}$.

We remark that in the proof of the above Theorem we have actually established the identity $\hat{b}(k)=\gamma(k) \nu(k+1) \nu(k)^{-1} b(k)$ for any $k \in \mathbb{N}^{*}$. Since the values $b(0)$ and $\hat{b}(0)$ does not have influence on the solutions of the corresponding equations, we can extend the identity to 0 . We always adopt this criteria, when necessary.

Proposition 5.3. Given $\nu \in \ell\left(\mathbb{K}^{*}\right)$, the equations (3) and (5) are $\nu$-equivalent iff

$$
\hat{g}(k, s)=\frac{\nu(k) a(s)}{\nu(s+1) \hat{a}(s)} g(k, s), \quad k, s \in \mathbb{N} .
$$

Proof. If the identity holds, then $\widehat{\mathcal{S}}=\nu \mathcal{S}$ since $\{\hat{g}(\cdot, s), \hat{g}(\cdot, s+1)\}$ and $\{g(\cdot, s) \gamma(s), g(\cdot, s+1) \gamma(s+1)\}$, where $\gamma(s)=\nu(s+1)^{-1} \hat{a}(s)^{-1} a(s)$ are basis of $\widehat{\mathcal{S}}$ and $\mathcal{S}$, respectively.

Conversely, given $\{z, w\}$ a basis of $\mathcal{S}$, applying Corollary 4.6 we have

$$
\hat{g}(k, s)=\frac{\nu(k) \nu(s)}{\hat{a}(s) \nu(s) \nu(s+1) \omega[z, w](s)}[w(k) z(s)-z(k) w(s)]=\frac{\nu(k) a(s)}{\hat{a}(s) \nu(s+1)} g(k, s),
$$

where we have taken into account that $\omega[\nu z, \nu w]=\nu \nu_{1} \omega[z, w]$.

In the very special case when $\hat{a}=c, \hat{b}=b$ and $\hat{c}=a$, equation (5) is called the adjoint equation to (3) and the space of its solutions and its Green function are denoted by $\mathcal{S}^{*}$ and $g^{*}$, respectively. Moreover, when $a=c$, equation (3) is called self-adjoint, since that (3) and (5) coincide. We also define the companion sequence of Equation (3) as $\rho \in \ell\left(\mathbb{K}^{*}\right)$ given by

$$
\rho(k)=\prod_{j=0}^{k-1} \frac{a(j)}{c(j)}, \quad k \in \mathbb{N} .
$$

The equation (3) is self-adjoint iff $\rho=1$. Moreover, since $\rho_{1} c=\rho a$, we obtain that equation (3) is equivalent to the self-adjoint equation with coefficients $\rho a$ and $\rho b$.

With the companion function we can nicely express the result of Proposition 4.4: For any $z, w \in \mathcal{S}$ the function $\rho a \omega[z, w]$ is constant and moreover this constant is non null iff $\{z, w\}$ is a basis of $\mathcal{S}$.

The relationship between solutions of a given homogeneous equations and its adjoint equation is shown in the following result.

Proposition 5.4. Given $a, c \in \ell\left(\mathbb{K}^{*}\right)$ and $b, \hat{b} \in \ell(\mathbb{K})$ if $z, w \in \ell(\mathbb{K})$ satisfy that

$$
a(k) z(k+1)-b(k) z(k)+c(k-1) z(k-1)=c(k) w(k+1)-\hat{b}(k) w(k)+a(k-1) w(k-1)=0, \quad k \in \mathbb{N}^{*},
$$


then for any $m, n \in \mathbb{N}$ such that $m<n$ we have,

$$
\begin{aligned}
\int_{m}^{n} \hat{b}(s) z(s) w(s) \nabla s & =\int_{m}^{n} b(s) z(s) w(s) \nabla s+a(n) \omega[z, w](n)-c(m) \omega[z, w](m) \\
& -(a(n)-c(n)) z(n) w(n+1)+(a(m)-c(m)) z(m+1) w(m) .
\end{aligned}
$$

Proof. We have the following identities

$$
\begin{aligned}
\int_{m}^{n} b(s) z(s) w(s) \nabla s & =\sum_{s=m+1}^{n} b(s) z(s) w(s)=\sum_{s=m+1}^{n} a(s) z(s+1) w(s)+\sum_{s=m+1}^{n} c(s-1) z(s-1) w(s) \\
& =\sum_{s=m+1}^{n} a(s-1) z(s) w(s-1)+\sum_{s=m+1}^{n} c(s) z(s) w(s+1) \\
& +a(n) z(n+1) w(n)-a(m) z(m+1) w(m)+c(m) z(m) w(m+1)-c(n) z(n) w(n+1) \\
& =\sum_{s=m+1}^{n}(\hat{b}(s) z(s) w(s))+c(m) \omega[z, w](m)-a(n) \omega[z, w](n) \\
& +(c(m)-a(m)) z(m+1) w(m)+(a(n)-c(n)) z(n) w(n+1) .
\end{aligned}
$$

Notice that when $\hat{b}=b$ and the equation is self-adjoint, then the identity in the above Proposition simply say that for any $z, w \in \mathcal{S}$, the sequence $a \omega[z, w]$ is constant.

Proposition 5.5. The Equation (3) is $\rho$-equivalent to its adjoint and moreover $g^{*}(k, s)=-g(s, k)$ for any $k, s \in \mathbb{N}$. In addition, $\rho(k) g(k, s)=-\rho(s) g(s, k)$ for any $k, s \in \mathbb{N}$ and hence a given equation is self-adjoint iff its Green function is skew-symmetric.

Proof. If $z \in \ell(\mathbb{K})$, defining $u=\rho z$, then $z \in \mathcal{S}$ iff

$$
\begin{aligned}
0 & =\rho(k)(a(k) z(k+1)-b(k) z(k)+c(k-1) z(k-1)) \\
& =c(k) \rho(k+1) z(k+1)-b(k) \rho(k) z(k)+a(k-1) \rho(k-1) z(k-1) \\
& =c(k) u(k+1)-b(k) u(k)+a(k-1) u(k-1)
\end{aligned}
$$

and hence iff $u \in \mathcal{S}^{*}$, which implies that $\mathcal{S}^{*}=\rho \mathcal{S}$. Applying now the Proposition 5.3 we have

$$
g^{*}(k, s)=\frac{\rho(k) a(s)}{c(s) \rho(s+1)} g(k, s)=\frac{\rho(k)}{\rho(s)} g(k, s), \quad k, s \in \mathbb{N} .
$$

On the other hand, if $\{z, w\}$ is a basis of $\mathcal{S}$, then $\{\rho z, \rho w\}$ is a basis of $\mathcal{S}^{*}$ and hence applying Corollary 4.6

$$
\begin{aligned}
g^{*}(k, s) & =\frac{\rho(k) \rho(s)}{c(s) \omega[\rho z, \rho w](s)}[w(k) z(s)-z(k) w(s)]=\frac{\rho(k)}{c(s) \rho(s+1) \omega[z, w](s)}[w(k) z(s)-z(k) w(s)] \\
& =\frac{\rho(k)}{a(s) \rho(s) \omega[z, w](s)}[w(k) z(s)-z(k) w(s)]=\frac{1}{a(k) \omega[z, w](k)}[w(k) z(s)-z(k) w(s)]=-g(s, k),
\end{aligned}
$$

where we have taken into account that $\rho a w[z, w]$ is constant. Finally, combining the two obtained expressions for $g^{*}$, we have that $-\rho(s) g(s, k)=\rho(s) g^{*}(k, s)=\rho(k) g(k, s)$ for any $k, s \in \mathbb{N}$. Therefore, if the 
equation is self-adjoint; that is if $\rho=1$, then $g$ is skew-symmetric. Conversely, if $g$ is skew-symmetric, then we have that $a(s)^{-1}=g(s+1, s)=-g(s, s+1)=c(s)^{-1}$ for any $s \in \mathbb{N}$ and hence the equation is self-adjoint.

The coefficients of the Equation (3) completely determine the space of its solutions $\mathcal{S}$ and hence its Green function and the solutions of any initial value problem for any data. Since the Green function determines the first coefficient of the equation and also the space $\mathcal{S}$ we conclude that Equation (3) and hence the solution of the Equation (2) for any data are completely determined by the Green function. Moreover, structural properties of the equation, such as its self-adjointness can be also read from its Green function.

We end this section glueing most of the above results to provide a characterization of those double sequences $g: \mathbb{N} \times \mathbb{N} \longrightarrow \mathbb{K}$ that are the Green function for some linear difference equation of second order. Moreover, we explicitly determine the coefficients of that equation from $g$.

Theorem 5.6. A double sequence $g: \mathbb{N} \times \mathbb{N} \longrightarrow \mathbb{K}$ is the Green function of some second order difference equation iff it satisfies the following properties:

(i) $g(k+1, k) \neq 0$, for any $k \in \mathbb{N}$.

(ii) $\omega[g(\cdot, 0), g(\cdot, 1)] \in \ell\left(\mathbb{K}^{*}\right)$.

(iii) $g(k, s)=\frac{g(s+1, s)}{\omega[g(\cdot, 0), g(\cdot, 1)](s)}[g(k, 1) g(s, 0)-g(k, 0) g(s, 1)]$, for any $k, s \in \mathbb{N}$.

If $g$ satisfies the above properties, then $g(k, k)=0$ and $g(k, k+1) \neq 0$ for any $k \in \mathbb{N}$ and moreover, $g$ is the Green function of the equation $a(k) x(k+1)-b(k) x(k)+c(k-1) x(k-1)=0, k \in \mathbb{N}^{*}$, where the coefficients $a, b, c \in \ell(K)$ are given by

$$
a(k)=\frac{1}{g(k+1, k)}, \quad b(k)=\frac{g(k+1, k-1)}{g(k+1, k) g(k, k-1)}, \quad c(k)=\frac{-1}{g(k, k+1)}, \quad k \in \mathbb{N} .
$$

Proof. Assume that $g$ is the Green function of the equation $a(k) x(k+1)-b(k) x(k)+c(k-1) x(k-1)=0$, $k \in \mathbb{N}^{*}$. Then, $g(k+1, k), g(k, k+1) \neq 0$ and moreover from (4) we have the expression for $a, b$ and $c$ in terms of the Green function. In addition from Corollary $4.5,\{g(\cdot, s), g(\cdot, s+1)\}$ is a basis for any $s \in \mathbb{N}$, and hence (ii) is also true and moreover we get (iii) from Corollary 4.6.

Conversely, if properties (i) to (iii) are satisfied, then taking $s=k$ in (iii) we obtain that $g(k, k)=0$, whereas taking $s=k+1$ we obtain that

$$
g(k, k+1)=-\frac{g(k+2, k+1) \omega[g(\cdot, 0), g(\cdot, 1)](k)}{\omega[g(\cdot, 0), g(\cdot, 1)](k+1)} \neq 0 .
$$

In addition, if we consider $x=g(\cdot, 0)$ and $y=g(\cdot, 1)$ since for any $k \in \mathbb{N}^{*}$ from (iii) we have that

$$
\frac{b(k)}{a(k)}=\frac{[y(k+1) x(k-1)-x(k+1) y(k-1)]}{\omega[x, y](k-1)} \text { and } \frac{c(k-1)}{a(k)}=\frac{\omega[x, y](k)}{\omega[x, y](k-1)}
$$

then

$$
x(k+1)-\frac{b(k)}{a(k)} x(k)+\frac{c(k-1)}{a(k)} x(k-1)=y(k+1)-\frac{b(k)}{a(k)} y(k)+\frac{c(k-1)}{a(k)} y(k-1)=0 .
$$

Therefore, if we consider the sequence $z(k)=g(k, s), k \in \mathbb{N}$, where $s \in \mathbb{N}$ is fixed, then from (iii) $z$ is a linear combination of $x$ and $y$ and hence it satisfies that

$$
a(k) z(k+1)-b(k) z(k)+c(k-1) z(k-1)=0, \quad k \in \mathbb{N}^{*} .
$$


The result follows bearing in mind that $z(s)=0$ and moreover $z(s+1)=a(s)^{-1}$.

\section{Reduction of order}

In this section we develop a simple technique that allows us to obtain the Green function of a linear second order difference equation when an always non-null solution of the homogeneous equation is known. Since we only need to known another linearly independent solution, we call this method reduction of order, that in fact consists in a transformation of the given homogeneous equation into the discrete version of a linear ODE of the form $\left(\alpha(t) x^{\prime}(t)\right)^{\prime}=0$, that can be solved by integrating twice.

Given $a, c \in \ell\left(\mathbb{K}^{*}\right)$ and $b \in \ell(\mathbb{K})$, assume that a solution, say $\nu$, of the Equation (3) is known and moreover that $\nu \in \ell\left(\mathbb{K}^{*}\right)$. Therefore, we have that

$$
b(k)=\frac{1}{\nu(k)}[a(k) \nu(k+1)+c(k-1) \nu(k-1)], \quad k \in \mathbb{N}^{*}
$$

and then, $z \in \ell(\mathbb{K})$ is a solution of (3) iff

$$
\begin{aligned}
0 & =a(k) z(k+1)-\frac{z(k)}{\nu(k)}[a(k) \nu(k+1)+c(k-1) \nu(k-1)]+c(k-1) z(k-1) \\
& =a(k) \nu(k+1)\left(\frac{z(k+1)}{\nu(k+1)}-\frac{z(k)}{\nu(k)}\right)+c(k-1) \nu(k-1)\left(\frac{z(k-1)}{\nu(k-1)}-\frac{z(k)}{\nu(k)}\right), \quad k \in \mathbb{N}^{*} .
\end{aligned}
$$

If we consider now $\rho$ the companion sequence and multiply both sides of the above identity by $\nu \rho$, we obtain that $z$ is a solution of (3) iff $\nu^{-1} z$ is a solution of the self-adjoint equation

$$
0=\rho(k) a(k) \nu(k) \nu(k+1)(x(k+1)-x(k))+\rho(k-1) a(k-1) \nu(k) \nu(k-1)(x(k-1)-x(k)) .
$$

So, we have proved the following result.

Lemma 6.1. Given $a, c \in \ell\left(\mathbb{K}^{*}\right)$ and $b \in \ell(\mathbb{K})$, if the equation

$$
a(k) z(k+1)-b(k) z(k)+c(k-1) z(k-1)=0, \quad k \in \mathbb{N}^{*}
$$

has a solution $\nu \in \ell\left(\mathbb{K}^{*}\right)$, then it is $\nu$-equivalent to the self-adjoint equation

$$
\hat{a}(k) x(k+1)-(\hat{a}(k)+\hat{a}(k-1)) x(k)+\hat{a}(k-1) x(k-1)=0, \quad k \in \mathbb{N}^{*},
$$

where $\hat{a}=\rho a \nu \nu_{1}$.

Next, we describe the reduction of order technique. Given $x \in \ell(\mathbb{K})$ and defining $X \in \ell(\mathbb{K})$ as

$$
X(k)=\hat{a}(k)(x(k+1)-x(k)), \quad k \in \mathbb{N},
$$

we have that $x$ satisfies that $\hat{a}(k) x(k+1)-(\hat{a}(k)+\hat{a}(k-1)) x(k)+\hat{a}(k-1) x(k-1)=0, k \in \mathbb{N}^{*}$ iff

$$
X(k)-X(k-1)=0, \quad k \in \mathbb{N}^{*},
$$

which implies that $X$ is constant. So, $x$ satisfies that $\hat{a}(k) x(k+1)-(\hat{a}(k)+\hat{a}(k-1)) x(k)+\hat{a}(k-1) x(k-1)=0$, 
$k \in \mathbb{N}^{*}$ iff there exists $\beta \in \mathbb{K}$ such that

$$
x(k)-x(k-1)=\frac{\beta}{\hat{a}(k-1)}, \quad k \in \mathbb{N}^{*} .
$$

Applying the Fundamental Theorem of Calculus we conclude that $x$ is a solution of the above equation iff there exist $\alpha, \beta \in \mathbb{K}$ such that

$$
x(k)=\alpha+\beta \int_{0}^{k} \frac{\nabla s}{\hat{a}(s-1)}, \quad k \in \mathbb{N} .
$$

Theorem 6.2. Consider $a, c \in \ell\left(\mathbb{K}^{*}\right), b \in \ell(\mathbb{K})$ and the difference equation

$$
a(k) z(k+1)-b(k) z(k)+c(k-1) z(k-1)=0, \quad k \in \mathbb{N}^{*} .
$$

If $\nu \in \ell\left(\mathbb{K}^{*}\right)$ is a solution, then the Green function is given by

$$
g(k, s)=\nu(k) \nu(s) \rho(s) \int_{s}^{k} \frac{\nabla r}{\nu(r) \nu(r-1) \rho(r-1) a(r-1)}, \quad \text { for any } k, s \in \mathbb{N}
$$

Proof. If $\zeta \in \ell(\mathbb{K})$ is defined as

$$
\zeta(k)=\nu(k) \int_{0}^{k} \frac{\nabla s}{\nu(s) \nu(s-1) \rho(s-1) a(s-1)}, \quad k \in \mathbb{N}
$$

then $\zeta(0)=0, \zeta(1)=a(0)^{-1} \nu(0)^{-1}$ and hence, $\omega[\nu, \zeta](0)=a(0)^{-1}$. Therefore, the previous reasoning shows that $\zeta$ is a solution of the equation $a(k) z(k+1)-b(k) z(k)+c(k-1) z(k-1)=0, k \in \mathbb{N}^{*}$, and we obtain that $\{\nu, \zeta\}$ is a basis of the space of its solutions. Then, the result follows applying Corollary 4.6 and taking into account that $\rho a \omega[z, \zeta]$ is constant and hence equal to 1 , since $\rho(0) a(0) \omega[z, \zeta](0)=1$.

We remark that we can use the above technique to solve a wide class of equations. Specifically, if we fix $a, c \in \ell\left(\mathbb{K}^{*}\right)$ and consider the map $\mathcal{B}_{a, c}: \ell\left(\mathbb{K}^{*}\right) \longrightarrow \ell(\mathbb{K})$ defined by

$$
\mathcal{B}_{a, c}(\nu)(0)=\frac{a(0) \nu(1)}{\nu(0)}, \quad \mathcal{B}_{a, c}(\nu)(k)=\frac{1}{\nu(k)}[a(k) \nu(k+1)+c(k-1) \nu(k-1)], \quad k \in \mathbb{N}^{*},
$$

we can obtain the Green function for any equation $a(k) z(k+1)-b(k) z(k)+c(k-1) z(k-1)=0, k \in \mathbb{N}^{*}$ such that $b \in \operatorname{Img} \mathcal{B}_{a, c}$. This happens, for instance when $b(k)=a(k)+c(k-1)$ for any $k \in \mathbb{N}^{*}$, since then $b=\mathcal{B}_{a, c}(1)$. It is clear that the map $\mathcal{B}_{a, c}$ is not injective: If $\lambda \in \mathbb{K}$, then $\mathcal{B}_{a, c}(\lambda \nu)=\mathcal{B}_{a, c}(\nu)$ for any $\nu \in \ell\left(\mathbb{K}^{*}\right)$.

More generally, let $b \in \operatorname{Img} \mathcal{B}_{a, c}$ and $\nu \in \ell\left(\mathbb{K}^{*}\right)$ such that $b=\mathcal{B}_{a, c}(\nu)$. Then, $\mu \in \ell\left(\mathbb{K}^{*}\right)$ satisfies that $b=\mathcal{B}_{a, c}(\mu)$ iff $a(0) \nu(0)^{-1} \nu(1)=b(0)=a(0) \mu(0)^{-1} \mu(1)$ and it is a solution of the homogeneous equation $a(k) z(k+1)-b(k) z(k)+c(k-1) z(k-1)=0, k \in \mathbb{N}^{*}$.

Therefore, with the previous notation, if $b=\mathcal{B}_{a, c}(\mu)$, then there exist $\alpha, \beta \in \mathbb{K}$ such that $\mu=\alpha \nu+\beta \zeta$ which, since $\zeta(0)=0$ implies that $\mu(0)=\alpha \alpha(0)$ and hence $\mu(1)=\nu(0)^{-1} \mu(0) \nu(1)=\alpha \nu(1)$. In conclusion, $\mu \in \ell\left(\mathbb{K}^{*}\right)$ satisfies that $b=\mathcal{B}_{a, c}(\mu)$ iff there exists $\alpha \in \mathbb{K}^{*}$ such that $\mu=\alpha \nu$.

To avoid this lack of injectivity, we consider the set $L\left(\mathbb{K}^{*}\right)=\left\{\nu \in \ell\left(\mathbb{K}^{*}\right): \nu(0)=1\right\}$. Then, we have proved the following result.

Proposition 6.3. For any $a, c \in \ell\left(\mathbb{K}^{*}\right)$ the map $\mathcal{B}_{a, c}$ is injective on $L\left(\mathbb{K}^{*}\right)$. 
As a non trivial example of reduction of order we consider the Hermite difference equation

$$
z(k+1)-2 x z(k)+2 k z(k-1)=0, \quad k \in \mathbb{N}^{*},
$$

where $x \in \mathbb{R}, a(k)=1, b(k)=2 x$ and $c(k)=2(k+1)$ for any $k \in \mathbb{N}$. It is known that

$$
H_{k}(x)=\sum_{s=0}^{\left\lfloor\frac{k}{2}\right\rfloor}(-1)^{s} \frac{k !(2 x)^{k-2 s}}{s !(k-2 s) !}, \quad k \in \mathbb{N},
$$

is a solution of the Hermite equation (6), see for instance [10, 17], that as a function of the variable $x, H_{k}$ is known as $k$-th Hermite polynomial. Since the set of zeroes of the sequence $\left\{H_{0}(x)\right\}_{k=0}^{\infty}$ is countable, we can choose $x \in \mathbb{R}$ such that $H_{k}(x) \neq 0$, for any $k \in \mathbb{N}^{*}(x=1$ is a value with this property).

The companion function for the Hermite equation (6) is $\rho(s)=\frac{1}{2^{s} s !}$ and hence the Green function for the Hermite equation is

$$
g(k, s)=\operatorname{sign}(k-s) \sum_{r=\min \{k, s\}}^{\max \{k, s\}+1} 2^{r} r ! \frac{H_{k}(x) H_{s}(x)}{H_{r}(x) H_{r+1}(x)}, \text { for any } k, s \in \mathbb{N} .
$$

We conclude this section by introducing a very particular case. The difference Equation (3) is called exact when any constant sequence is a solution. Equivalently, Equation (3) is exact iff $b(k)=a(k)+c(k-1)$ for any $k \in \mathbb{N}$.

Corollary 6.4. The Equation (3) is exact iff its Green function is given by

$$
g(k, s)=\rho(s) \int_{s}^{k} \frac{\nabla r}{\rho(r-1) a(r-1)}, \text { for any } k, s \in \mathbb{N} .
$$

Proof. If the equation is exact, the expression for the Green function is consequence of Theorem 6.2. Conversely, from Identity (4) we have

$b(k)=a(k) a(k-1) g(k+1, k-1)=a(k) a(k-1) \rho(k-1)\left[\frac{1}{\rho(k-1) a(k-1)}+\frac{1}{a(k) \rho(k)}\right]=a(k)+c(k-1)$,

for any $k \in \mathbb{N}$.

As a simple example we can consider the case of exact equation with linear coefficients, that appears in enumerative combinatorics, see [10]. So, if $a(k)=\alpha(k+n), c(k)=\beta(k+m)$ where $\alpha, \beta \neq 0$ and $n, m \in \mathbb{N}^{*}$, then the Green function of the difference equation

$$
\alpha(k+n) z(k+1)-((\alpha+\beta) k+\alpha n+\beta(m-1)) z(k)+\beta(k+m-1) z(k-1)=0
$$

is given by

$$
g(k, s)=\alpha^{-1} \operatorname{sign}(k-s) \frac{(s+n-1) !}{(s+m-1) !} \sum_{r=\min \{k, s\}}^{\max \{k, s\}+1}\left(\frac{\alpha}{\beta}\right)^{s-r} \frac{(r+m-1) !}{(r+n) !}, \text { for any } k, s \in \mathbb{N} .
$$


The fact that we know the solutions of any exact equation allows us to obtain, from Proposition 5.4, a nice and useful identity about the weighted integral of a solution of a homogeneous difference equation.

Proposition 6.5. Given $z \in \mathcal{S}$, for any $m, n \in \mathbb{N}$ such that $m<n$ the following identities are satisfied:

$$
\begin{aligned}
\int_{m}^{n}[a(s-1)+c(s)-b(s)] z(s) \nabla s & =c(n) z(n)-a(n) z(n+1)+a(m) z(m+1)-c(m) z(m) \\
\int_{m}^{n}[a(s-1)+c(s)-b(s)] z(s) \zeta^{*}(s) \nabla s & =a(n) \omega\left[z, \zeta^{*}\right](n)-c(m) \omega\left[z, \zeta^{*}\right](m) \\
& -(a(n)-c(n)) z(n) \zeta^{*}(n+1)+(a(m)-c(m)) z(m+1) \zeta^{*}(m),
\end{aligned}
$$

where

$$
\zeta^{*}(k)=\int_{0}^{k} \frac{\rho(s-1) \nabla s}{c(s-1)}, \quad k \in \mathbb{N} .
$$

\section{Chebyshev Functions}

Fixed $a, c \in \ell\left(\mathbb{K}^{*}\right)$ and $b \in \ell(\mathbb{K})$, Theorem 4.3 establishes that to solve any initial value problem with any data for a difference equation with coefficients $a, b$ and $c$ it suffices to compute the corresponding Green function. To achieve this goal, it is useful to introduce some previous concepts and notations with the same spirit than in [7] but with slight modifications, since the Green function does not depend on $a(0)$ and $b(0)$.

Given $p \in \mathbb{N}^{*}$, a multi-index of order $p$ is a $p$-tuple $\alpha=\left(\alpha_{1}, \ldots, \alpha_{p}\right) \in \mathbb{N}^{p}$. Given $\alpha=\left(\alpha_{1}, \ldots, \alpha_{p}\right) \in$ $\mathbb{N}^{p}$ its length is defined as $|\alpha|=\sum_{j=1}^{p} \alpha_{j}$ and its shift is $\tilde{\alpha}$ the multi-index of order $p$ defined as $\tilde{\alpha}=$ $\left(0, \alpha_{1}, \ldots, \alpha_{p-1}\right)$. Moreover $0_{p}$ and $\pi_{p}$ denote the multi-indexes of order $p$ defined as $0_{p}=(0, \ldots, 0)$ and as $\pi_{p}=(1, \ldots, 1)$, respectively.

A binary multi-index of order $p$ is a $p$-tuple $\alpha=\left(\alpha_{1}, \ldots, \alpha_{p}\right) \in\{0,1\}^{p}$. The set of binary multi-indexes of order $p$ is denoted by $\ell_{p}$. Clearly, $0_{p}, \pi_{p} \in \ell_{p}, \operatorname{card}\left(\ell_{p}\right)=2^{p}$, for any $p \in \mathbb{N}^{*}$ and moreover, given $\alpha \in \ell_{p}$ then $\tilde{\alpha} \in \ell_{p}$.

Given $\alpha \in \ell_{p}$, then $0 \leq|\alpha| \leq p$ and $|\alpha|=m$ iff exactly $m$ components of $\alpha$ are equal to 1 and exactly $p-m$ components of $\alpha$ are equal to 0 . Therefore, $0_{p}$ is the unique binary multi-index of order $p$ and null length, whereas the unique binary multi-index of order $p$ and length equals to $p$ is $\pi_{p}$. Moreover, if $|\alpha|=m \geq 1$, we denote by $i_{1}, \ldots, i_{m}$ the indexes such that $1 \leq i_{1}<\cdots<i_{m} \leq p$ and $\alpha_{i_{j}}=1$, $j=1, \ldots, m$.

For any $p \in \mathbb{N}^{*}$, we are interested only on those binary multi-indexes of order $p$ whose length is $\left\lfloor\frac{p}{2}\right\rfloor$ at most. Next, we define these sets of binary multi-indexes:

(i) For $p \in \mathbb{N}^{*}, \ell_{p}^{0}=\left\{\alpha \in \ell_{p}:|\alpha|=0\right\}=\left\{0_{p}\right\}=\{(0, \ldots, 0)\}$.

(ii) For $p \geq 2, \ell_{p}^{1}=\left\{\alpha \in \ell_{p}: \alpha_{p}=0\right.$ and $\left.|\alpha|=1\right\}$.

(iii) For $p \geq 4$ and $m=2, \ldots,\left\lfloor\frac{p}{2}\right\rfloor, \ell_{p}^{m}=\left\{\alpha \in \ell_{p}: \alpha_{p}=0,|\alpha|=m\right.$ and $\left.i_{j+1}-i_{j} \geq 2, j=1, \ldots, m-1\right\}$.

Note that for any $m \in \mathbb{N}^{*}$, we have $\ell_{2 m}^{m}=\{(1,0,1, \ldots, 0,1,0)\}$. Moreover, if $p \in \mathbb{N}^{*}$ and $m=0, \ldots,\left\lfloor\frac{p}{2}\right\rfloor$, then $|\tilde{\alpha}|=m$ for any $\alpha \in \ell_{p}^{m}$.

Clearly, $\operatorname{card}\left(\ell_{p}^{0}\right)=1$ for any $p \in \mathbb{N}^{*}$ and $\operatorname{card}\left(\ell_{p}^{1}\right)=p-1$ for any $p \geq 2$. On the other hand if $p \geq 4$, 
since choosing $m$ locations for the ones in $\alpha \in \ell_{p}^{m}, m=2, \ldots,\left\lfloor\frac{p}{2}\right\rfloor$, implies fix other $m-1$ locations with zeroes between $i_{1}$ an $i_{m}$, we can choose $m$ locations among $p-1-(m-1)$ available, which implies that $\operatorname{card}\left(\ell_{p}^{m}\right)=\left(\begin{array}{c}p-m \\ m\end{array}\right)$. Moreover, this formula also works for $\operatorname{card}\left(\ell_{p}^{0}\right), p \in \mathbb{N}^{*}$, and for $\operatorname{card}\left(\ell_{p}^{1}\right), p \geq 2$.

Given $p \in \mathbb{N}^{*}$ and $m=0, \ldots,\left\lfloor\frac{p}{2}\right\rfloor$, for any $\alpha \in \ell_{p}^{m}$, its complementary is $\bar{\alpha}=\left(\bar{\alpha}_{1}, \ldots, \bar{\alpha}_{p}\right) \in \ell_{p}$, the binary multi-index of order $p$ defined as

$$
\bar{\alpha}_{i_{j}}=\bar{\alpha}_{i_{j}+1}=0, \quad j=1, \ldots, m \text { and } \bar{\alpha}_{i}=1, \quad i=1, \ldots, p, \quad i \neq i_{j}, i_{j}+1, \quad j=1, \ldots, m .
$$

It is clear that $|\bar{\alpha}|=p-2 m$. In particular, if $\alpha=0_{p}, p \in \mathbb{N}^{*}$, then $\bar{\alpha}=\pi_{p}$, whereas for any $m \in \mathbb{N}^{*}$ if $\alpha \in \ell_{2 m}^{m}$, then $\bar{\alpha}=0_{p}$. More generally, given $p \in \mathbb{N}^{*}$ and $m=0, \ldots,\left\lfloor\frac{p}{2}\right\rfloor$, we have the following relation between a binary multi-index in $\ell_{p}^{m}$ and its complementary:

$$
\pi_{p}=\alpha+\bar{\alpha}+\tilde{\alpha}, \text { for any } \alpha \in \ell_{p}^{m} .
$$

The relation between the sets $\ell_{p+1}^{m}, \ell_{p}^{m}$ and $\ell_{p-1}^{m-1}$ is given in the following result, whose proof is straightforward.

Lemma 7.1. The following identities hold:

(i) For any $p \geq 2$ and any $m=1, \ldots,\left\lfloor\frac{p}{2}\right\rfloor$ then

$$
\ell_{p+1}^{m}=\left(\ell_{p}^{m} \times\{0\}\right) \cup\left(\ell_{p-1}^{m-1} \times\{(1,0)\}\right)=\left(\{0\} \times \ell_{p}^{m}\right) \cup\left(\{(1,0)\} \times \ell_{p-1}^{m-1}\right) .
$$

(ii) If $p \geq 1$ is odd, then $\ell_{p+1}^{\left\lfloor\frac{p+1}{2}\right\rfloor}=\ell_{p-1}^{\left\lfloor\frac{p-1}{2}\right\rfloor} \times\{(1,0)\}=\{(1,0)\} \times \ell_{p-1}^{\left\lfloor\frac{p-1}{2}\right\rfloor}$.

(iii) If $\alpha=(\beta, 0)$ with $\beta \in \ell_{p}^{m}$, then $\bar{\alpha}=(\bar{\beta}, 1)$, whereas if $\alpha=(\beta, 1,0)$ with $\beta \in \ell_{p-1}^{m-1}$, then $\bar{\alpha}=(\bar{\beta}, 0,0)$. Analogously, if $\alpha=(0, \beta)$ with $\beta \in \ell_{p}^{m}$, then $\bar{\alpha}=(1, \bar{\beta})$, whereas if $\alpha=(1,0, \beta)$ with $\beta \in \ell_{p-1}^{m-1}$, then $\bar{\alpha}=(0,0, \bar{\beta})$.

Given $x \in \ell(\mathbb{K})$, for any $p \in \mathbb{N}^{*}$ and any multi-index $\alpha=\left(\alpha_{1}, \ldots, \alpha_{p}\right) \in \mathbb{N}^{p}$ we define

$$
x^{\alpha}=\prod_{j=1}^{p} x(j)^{\alpha_{j}} .
$$

In particular $x^{0_{p}}=1$ and $x^{\pi_{p}}=x(1) \cdots x(p)$. For the sake of completeness, we also define $x^{\pi_{0}}=1$ and when $x(0) \neq 0, x^{\pi_{-1}}=x(0)^{-1}$.

If $x, y \in \ell(\mathbb{K})$ and $\alpha \in \mathbb{N}^{p}$, then $(x y)^{\alpha}=x^{\alpha} y^{\alpha}$. Moreover, $x^{\alpha+\beta}=x^{\alpha} x^{\beta}$ for any $\alpha, \beta \in \mathbb{N}^{p}$ and

$$
x^{\tilde{\alpha}}=\left(x_{1}\right)^{\alpha}, \text { for any } \alpha \in \ell_{p}^{m}, m=0, \ldots,\left\lfloor\frac{p}{2}\right\rfloor .
$$

If $x \in \ell\left(\mathbb{K}^{*}\right)$, for any multi-indexes $\alpha, \beta \in \mathbb{N}^{p}$ we also define

$$
x^{\alpha-\beta}=\prod_{j=1}^{p} x(j)^{\alpha_{j}-\beta_{j}}=x^{\alpha}\left(x^{\beta}\right)^{-1}
$$

and, in particular, $x^{-\beta}=\left(x^{\beta}\right)^{-1}=\left(x^{-1}\right)^{\beta}$, which implies that $x^{\alpha-\beta}=x^{\alpha} x^{-\beta}$. Therefore, for any $1 \leq s \leq p$ 
we have that $x^{\pi_{p}-\pi_{s-1}}=\prod_{j=s}^{p} x(j)$ and hence, the solutions of any homogeneous first order difference equation and its Green function can be easily expressed with this notation.

Observe that given $\alpha \in \mathbb{N}^{p}$, for $x \in \ell(\mathbb{K})$ and $y \in \ell\left(\mathbb{K}^{*}\right), x^{\alpha}$ and $y^{-\alpha}$ do not take into account the values $x(0)$ and $y(0)$. With this notation, the companion function associated to the coefficients $a, c \in \ell\left(\mathbb{K}^{*}\right)$ can be expressed as

$$
\rho(k)=\prod_{j=0}^{k-1} \frac{a(j)}{c(j)}=a(0) c(0)^{-1} a^{\pi_{k-1}} c^{-\pi_{k-1}}, \quad k \in \mathbb{N} .
$$

For any $k \in \mathbb{N}^{*}$, we define the $k$-th Chebyshev function on $\ell(\mathbb{K})$, the function $P_{k}: \ell(\mathbb{K}) \times \ell(\mathbb{K}) \longrightarrow \mathbb{K}$ given by

$$
P_{k}(x, y)=\sum_{m=0}^{\left\lfloor\frac{k}{2}\right\rfloor}(-1)^{m} \sum_{\alpha \in \ell_{k}^{m}} x^{\bar{\alpha}} y^{\alpha}, \quad x, y \in \ell(\mathbb{K}) .
$$

We also define $P_{0}(x, y)=1$ and $P_{-1}(x, y)=0$. In addition, for any $x \in \ell(\mathbb{K})$ and $y \in \ell\left(\mathbb{K}^{*}\right)$ we define $P_{-2}\left(x_{1}, y_{1}\right)=-y(0)^{-1}$.

As we will show in the next section, the name of Chebyshev function for (8) is justified because its relation with the usual (one variable) Chebyshev polynomials of second kind.

Notice that for any $k \in \mathbb{N}$ the function $P_{k}$ does not take into account the values of the sequences at 0 and hence if $\hat{x}, \hat{y} \in \ell(\mathbb{K})$ are such that $\hat{x}_{1}=x_{1}, \hat{y}_{1}=y_{1}$, then $P_{k}(\hat{x}, \hat{y})=P_{k}(x, y)$.

Since for any $k \in \mathbb{N}^{*}$ we know that $\overline{0}_{p}=\pi_{k}$ and that if $\alpha=(0, \ldots, 0, \underbrace{1}_{j}, 0, \ldots, 0), j=1, \ldots, k-1$, then $\bar{\alpha}=(1, \ldots, 1, \underbrace{0}_{j}, 0,1, \ldots, 1)$ the $k$-th Chebyshev function can be re-write as

$$
P_{k}(x, y)=\prod_{i=1}^{k} x(i)-\sum_{j=1}^{k-1} y(j) \prod_{\substack{i=1 \\ i \neq j, j+1}}^{k} x(i)+\sum_{m=2}^{\left\lfloor\frac{k}{2}\right\rfloor}(-1)^{m} \sum_{\alpha \in \ell_{k}^{m}} x^{\bar{\alpha}} y^{\alpha}, \quad x, y \in \ell(\mathbb{K})
$$

and hence $P_{1}(x, y)=x(1), P_{2}(x, y)=x(1) x(2)-y(1), P_{k}(x, 0)=x^{\pi_{k}}$, for any $k \in \mathbb{N}$ and moreover

$$
P_{2 k-1}(0, y)=0 \quad \text { and } \quad P_{2 k}(0, y)=(-1)^{k} \prod_{j=1}^{k} y(2 j-1)
$$

since when $k \geq 1$ then, $\alpha \in \ell_{k}^{m}, m=0, \ldots,\left\lfloor\frac{k}{2}\right\rfloor$ is such that $\bar{\alpha}=0_{p}$ iff $k$ is even and $m=\frac{k}{2}$.

Lemma 7.2. Given $x, y \in \ell(\mathbb{K})$ and $z \in \ell\left(\mathbb{K}^{*}\right)$, then

$$
P_{k}(z x, z y)=z^{\pi_{k}} P_{k}\left(x, z_{1}^{-1} y\right), \quad \text { for any } k \in \mathbb{N} .
$$

Proof. The identity is trivial for $k=0$. Moreover, for any $k \in \mathbb{N}^{*}$, any $m=0, \ldots,\left\lfloor\frac{k}{2}\right\rfloor$ and any $\alpha \in \ell_{k}^{m}$, then

$$
(z x)^{\bar{\alpha}}(z y)^{\alpha}=z^{\bar{\alpha}} z^{\alpha} x^{\bar{\alpha}} y^{\alpha}=z^{\bar{\alpha}+\alpha} x^{\bar{\alpha}} y^{\alpha}=z^{\pi_{k}-\tilde{\alpha}} x^{\bar{\alpha}} y^{\alpha}=z^{\pi_{k}} z_{1}^{-\alpha} x^{\bar{\alpha}} y^{\alpha}=z^{\pi_{k}} x^{\bar{\alpha}}\left(z_{1}^{-1} y\right)^{\alpha}
$$


and the result follows.

Proposition 7.3. The Chebyshev functions are characterized by either of the two three-terms recurrences:

$$
\begin{array}{llll}
P_{k+1}(x, y)=x(k+1) P_{k}(x, y)-y(k) P_{k-1}(x, y), & k \in \mathbb{N}, & P_{-1}(x, y)=0, & P_{0}(x, y)=1, \\
P_{k+1}(x, y)=x(1) P_{k}\left(x_{1}, y_{1}\right)-y(1) P_{k-1}\left(x_{2}, y_{2}\right), & k \in \mathbb{N}, & P_{-1}(x, y)=0, & P_{0}(x, y)=1,
\end{array}
$$

for any $x, y \in \ell(\mathbb{K})$.

Proof. We prove that if $\left\{P_{k}\right\}_{k=0}^{\infty}$ is given by any of the two recurrences, then $P_{k}(x, y)=$ $\sum_{m=0}^{\left\lfloor\frac{k}{2}\right\rfloor}(-1)^{m} \sum_{\alpha \in \ell_{k}^{m}} x^{\bar{\alpha}} y^{\alpha}$, for any $k \in \mathbb{N}^{*}$. Moreover we only analyze the first recurrence, since the proof of the second one is similar.

For $k=0$, the right hand side of the recurrences gives $x(1) P_{0}(x, y)=x(1)$ and hence for $k=1$, the right side of the recurrence gives $x(2) P_{1}(x, y)-y(1) P_{0}(x, y)=x(1) x(2)-y(1)$. Therefore, the claim is true for $k=1,2$ and we prove the result by induction. If we assume the identities are true for any $1 \leq j \leq k$, $k \geq 2$, then

$$
x(k+1) P_{k}(x, y)-y(k) P_{k-1}(x, y)=A_{0}(x, y)+A_{k}(x, y)+B_{k}(x, y),
$$

where

$$
\begin{aligned}
& A_{0}(x, y)=x(k+1) \sum_{\beta \in \ell_{k}^{0}} x^{\bar{\beta}} y^{\beta}, \\
& A_{k}(x, y)=x(k+1) \sum_{m=1}^{\left\lfloor\frac{k}{2}\right\rfloor}(-1)^{m} \sum_{\beta \in \ell_{k}^{m}} x^{\bar{\beta}} y^{\beta}=\sum_{m=1}^{\left\lfloor\frac{k}{2}\right\rfloor}(-1)^{m} \sum_{\beta \in \ell_{k}^{m}} x(k+1) x^{\bar{\beta}} y^{\beta}, \\
& B_{k}(x, y)=y(k) \sum_{m=1}^{\left\lfloor\frac{k-1}{2}\right\rfloor+1}(-1)^{m} \sum_{\beta \in \ell_{k-1}^{m-1}} x^{\bar{\beta}} y^{\beta}=\sum_{m=1}^{\left\lfloor\frac{k-1}{2}\right\rfloor+1}(-1)^{m} \sum_{\beta \in \ell_{k-1}^{m-1}} x^{\bar{\beta}} y(k) y^{\beta} .
\end{aligned}
$$

First, since for any $k \geq 1$, if $\beta \in \ell_{k}^{0}=\{(0, \ldots, 0)\}$ then $\bar{\beta}=\pi_{k}$, we have

$$
A_{0}(x, y)=x(1) \cdots x(k) x(k+1)=\sum_{\alpha \in \ell_{k+1}^{0}} x^{\bar{\alpha}} y^{\alpha} .
$$

On the other hand, from part (iii) of Lemma 7.1, we have that

$$
A_{k}(x, y)=\sum_{m=1}^{\left\lfloor\frac{k}{2}\right\rfloor}(-1)^{m} \sum_{\alpha \in \ell_{k}^{m} \times\{0\}} x^{\bar{\alpha}} y^{\alpha} \quad \text { and } \quad B_{k}(x, y)=\sum_{m=1}^{\left\lfloor\frac{k-1}{2}\right\rfloor+1}(-1)^{m} \sum_{\alpha \in \ell_{k-1}^{m-1} \times\{(1,0)\}} x^{\bar{\alpha}} y^{\alpha} .
$$

When $k$ is even then, $\left\lfloor\frac{k}{2}\right\rfloor=\left\lfloor\frac{k-1}{2}\right\rfloor+1=\left\lfloor\frac{k+1}{2}\right\rfloor$ and then, applying part (i) of Lemma 7.1 we have

$$
A_{k}(x, y)+B_{k}(x, y)=\sum_{m=1}^{\left\lfloor\frac{k+1}{2}\right\rfloor}(-1)^{m} \sum_{\alpha \in \ell_{k+1}^{m}} x^{\bar{\alpha}} y^{\alpha}
$$


When $k$ is odd then, $\left\lfloor\frac{k}{2}\right\rfloor=\left\lfloor\frac{k-1}{2}\right\rfloor=\left\lfloor\frac{k+1}{2}\right\rfloor-1$ and then, applying parts (i) and (ii) of Lemma 7.1,

$$
A_{k}(x, y)+B_{k}(x, y)=\sum_{m=1}^{\left\lfloor\frac{k}{2}\right\rfloor}(-1)^{m} \sum_{\alpha \in \ell_{k+1}^{m}} x^{\bar{\alpha}} y^{\alpha}+(-1)^{\left\lfloor\frac{k+1}{2}\right\rfloor} \sum_{\alpha \in \ell_{k+1}^{\left\lfloor\frac{k+1}{2}\right.}} x^{\bar{\alpha}} y^{\alpha}=\sum_{m=1}^{\left\lfloor\frac{k+1}{2}\right\rfloor}(-1)^{m} \sum_{\alpha \in \ell_{k+1}^{m}} x^{\bar{\alpha}} y^{\alpha}
$$

In any case, the result follows.

Now we are ready to establish the main result in this section.

Theorem 7.4. Given $a, c \in \ell\left(\mathbb{K}^{*}\right)$ and $b \in \ell(\mathbb{K})$, then the Green function for the difference equation $a(k) z(k+1)-b(k) z(k)+c(k-1) z(k-1)=0, k \in \mathbb{N}^{*}$, is

$$
\begin{aligned}
g(k, s) & =(-1)^{\operatorname{sign}(k-s)} a^{-\pi_{k-1}} c^{-\pi_{s-1}}(a c)^{\pi_{\min \{k, s\}-1}} P_{|k-s|-1}\left(b_{\min \{k, s\}},(a c)_{\min \{k, s\}}\right) \\
& =a^{-\pi_{k-1}} c^{-\pi_{s-1}}\left[P_{k-2}\left(b_{1},(a c)_{1}\right) P_{s-1}(b, a c)-P_{k-1}(b, a c) P_{s-2}\left(b_{1},(a c)_{1}\right)\right], \quad k, s \in \mathbb{N} .
\end{aligned}
$$

Proof. Fixed $s \in \mathbb{N}$, consider $z \in \ell(\mathbb{K})$, the sequence defined as

$$
z(k)=\left\{\begin{array}{cc}
a^{\pi_{s-1}} a^{-\pi_{k-1}} P_{k-s-1}\left(b_{s}, a_{s} c_{s}\right), & k \geq s, \\
-c^{-\pi_{s-1}} c^{\pi_{k-1}} P_{s-k-1}\left(b_{k}, a_{k} c_{k}\right), & k \leq s .
\end{array}\right.
$$

Then, $z(s)=P_{-1}\left(b_{s},(a c)_{s}\right)=0$ and $z(s+1)=a(s)^{-1} P_{0}\left(b_{s},(a c)_{s+1}\right)=a(s)^{-1}$. Moreover, if $k>s$, then $k-1 \geq s$ and hence, applying Proposition 7.3

$$
\begin{aligned}
a(k) z(k+1) & =a^{\pi_{s-1}} a^{-\pi_{k-1}} P_{k-s}\left(b_{s},(a c)_{s}\right)=a^{\pi_{s-1}} a^{-\pi_{k-1}} b_{s}(k-s) P_{k-s-1}\left(b_{s},(a c)_{s}\right) \\
& -a^{\pi_{s-1}} a^{-\pi_{k-1}}(a c)_{s}(k-s-1) P_{k-s-2}\left(b_{s},(a c)_{s+1}\right)=b(k) z(k)-c(k-1) z(k-1) .
\end{aligned}
$$

Therefore, if $s=0$, then $z$ is a solution of the given homogeneous equations.

On the other hand, when $s \geq 1$ we also have

$$
z(s-1)=-c^{-\pi_{s-1}} c^{\pi_{s-2}} P_{0}\left(b_{s-1},(a c)_{s-1}\right)=-c(s-1)^{-1},
$$

which implies that $a(s) z(s+1)-b(s) z(s)+c(s-1) z(s-1)=0$. Therefore, if $s=1$, then $z$ is a solution of the given homogeneous equation. In addition, when $s \geq 2$ and $1 \leq k<s$, then $k+1 \leq s$ and hence, applying Proposition 7.3

$$
\begin{aligned}
c(k-1) z(k-1) & =-c^{-\pi_{s-1}} c^{\pi_{k-1}} P_{s-k}\left(b_{k-1},(a c)_{k-1}\right) \\
& =-b(k) c^{-\pi_{s-1}} c^{\pi_{k-1}} P_{s-k-1}\left(b_{k},(a c)_{k}\right)+(a c)(k) c^{-\pi_{s-1}} c^{\pi_{k-1}} P_{s-k-2}\left(b_{k+1},(a c)_{k+1}\right) \\
& =b(k) z(k)-a(k) z(k+1),
\end{aligned}
$$

and the first expression for the Green function follows.

Alternatively, if we consider $x=g(\cdot, 0)$ and $y=g(\cdot, 1)$, then $\omega[x, y](0)=a(0)^{-1} c(0)^{-1}$. Since $\{x, y\}$ is a basis of $\mathcal{S}$, taking into account that $a(s) \omega[x, y](s)=\rho(s)^{-1} a(0) \omega[x, y](0)$ and Identity (7), from Corollary 4.6 we obtain that

$$
g(k, s)=\frac{\rho(s)}{a(0) \omega[x, y](0)}[y(k) x(s)-x(k) y(s)]=a(0) a^{\pi_{s-1}} c^{-\pi_{s-1}}[y(k) x(s)-x(k) y(s)] .
$$


Since

$$
x(k)=a(0)^{-1} a^{-\pi_{k-1}} P_{k-1}(b, a c), \quad y(k)=a^{-\pi_{k-1}} P_{k-2}\left(b_{1},(a c)_{1}\right), \quad k \in \mathbb{N}^{*},
$$

we obtain the second identity for the Green function.

As an straightforward consequence of the above result we have the following identities for the Chebyshev functions

Corollary 7.5. Given $x \in \ell(\mathbb{K})$ and $y \in \ell\left(\mathbb{K}^{*}\right)$, we have

$$
P_{k-2}\left(x_{1}, y_{1}\right) P_{s-1}(x, y)-P_{k-1}(x, y) P_{s-2}\left(x_{1}, y_{1}\right)=(-1)^{\operatorname{sign}(k-s)} y^{\pi_{\min \{k, s\}-1}} P_{|k-s|-1}\left(x_{\min \{k, s\}}, y_{\min \{k, s\}}\right),
$$

for any $k, s \in \mathbb{N}$.

When $b=0$, the Equation (3) is named uncoupled. From the Equalities (9) we obtain the following result, that also we could have obtained directly.

Corollary 7.6. If $a, c \in \ell\left(\mathbb{K}^{*}\right)$, the Green function for the uncoupled equation

$$
a(k) z(k+1)+c(k-1) z(k-1)=0, \quad k \in \mathbb{N}^{*},
$$

is given by

$$
\begin{array}{rlrl}
g(2 k+1,2 s) & =(-1)^{|k-s|} a(2 s)^{-1}\left[\prod_{j=\min \{k, s\}+1}^{\max \{k, s\}} a(2 j)^{-1} c(2 j-1)\right]^{\operatorname{sign}(k-s)}, & & k, s \in \mathbb{N}, \\
g(2 k, 2 s+1) & =(-1)^{|k-s|-1} c(2 k)^{-1}\left[\prod_{j=\min \{k, s\}+1}^{\max \{k, s\}} a(2 j-1)^{-1} c(2 j)\right]^{\operatorname{sign}(k-s)}, & k, s \in \mathbb{N} \\
g(m, n) & =0, & & \text { otherwise. }
\end{array}
$$

Corollary 7.7. Given $a \in \ell\left(\mathbb{K}^{*}\right)$ and $b \in \ell(\mathbb{K})$, the Green function for the self-adjoint equation

$$
a(k) z(k+1)-b(k) z(k)+a(k-1) z(k-1)=0, \quad k \in \mathbb{N}^{*},
$$

it is given by

$$
\begin{aligned}
g(k, s) & =(-1)^{\operatorname{sign}(k-s)}\left(\prod_{j=\min \{k, s\}}^{\max \{k, s\}-1} a(j)\right)^{-1} P_{|k-s|-1}\left(b_{\min \{k, s\}}, a_{\min \{k, s\}}^{2}\right) \\
& =a^{-\pi_{k-1}} a^{-\pi_{s-1}}\left[P_{k-2}\left(b_{1}, a_{1}^{2}\right) P_{s-1}\left(b, a^{2}\right)-P_{k-1}\left(b, a^{2}\right) P_{s-2}\left(b_{1}, a_{1}^{2}\right)\right], \quad k, s \in \mathbb{N} .
\end{aligned}
$$

The equation $z(k+1)-b(k) z(k)+z(k-1)=0, k \in \mathbb{N}^{*}$, where $b \in \ell(\mathbb{R})$ is known as Harper equation with coefficient $b$. In particular, when $b(k)=E-\lambda \cos (2 \pi \omega k+\phi), E, \lambda, \omega \in \mathbb{R}, \phi \in[0,2 \pi)$ it is called quasiMathieu equation, whereas when $\omega \in \mathbb{Q}$ it is named Mathieu equation, see [3, 8]. There, the parameters $E, \lambda, \omega$ and $\phi$ are called energy, coupling, frequency and phase, respectively. Therefore, the Green function 
for the Harper equation with coefficient $b \in \ell(K)$ is

$$
g(k, s)=(-1)^{\operatorname{sign}(k-s)} \sum_{m=0}^{\left\lfloor\frac{\lfloor k-s \mid-1}{2}\right\rfloor}(-1)^{m} \sum_{\alpha \in \ell_{k}^{m}} \prod_{j=1}^{m} b(j+\min \{k, s\})^{\bar{\alpha}_{j}}, \quad k, s \in \mathbb{N} .
$$

In particular, the Green function for the quasi-Mathieu equation with null energy is

$$
g(k, s)=(-1)^{\operatorname{sign}(k-s)} \sum_{m=0}^{\left\lfloor\frac{|k-s|-1}{2}\right\rfloor} \lambda^{m} \sum_{\alpha \in \ell_{k}^{m}} \prod_{j=1}^{m} \cos \left(\bar{\alpha}_{j}(2 \pi \omega j+2 \pi \omega \min \{k, s\}+\phi)\right), \quad k, s \in \mathbb{N} .
$$

\section{Difference equations with constant coefficients and Chebyshev polynomials}

In this section we apply the results of Section 4 and Section 7 to the case in which the coefficients of the Equation (3) are constant. So, given $a, c \in \mathbb{K}^{*}$ and $b \in \mathbb{K}$, we consider the homogeneous difference equation with constant coefficients

$$
a x(k+1)-b x(k)+c x(k-1)=0, \quad k \in \mathbb{N}^{*}
$$

and as usual, denote by $\mathcal{S}$ the space of its solutions. Notice that the companion function for the Equation (10) is given by $\rho(k)=\left(c^{-1} a\right)^{k}, k \in \mathbb{N}$.

Equations with constant coefficients have deserved a lot of attention in recent times, see for instance [19] and references therein. There, given $\alpha, \beta, \tau, \xi \in \mathbb{R}, \xi \neq 0$, the generalized Horadam numbers, $\left\{H_{k}(\tau, \xi ; \alpha, \beta)\right\}_{k=0}^{\infty}$, were defined as the solution of the recurrence

$$
H_{k+1}=\tau H_{k}+\xi H_{k-1}, \quad k \in \mathbb{N}^{*}, \quad H_{0}=\alpha, H_{1}=\beta .
$$

So the solutions of Equation (10) correspond to the Horadam sequences $\left\{H_{k}\left(a^{-1} b,-a^{-1} c ; \alpha, \beta\right)\right\}_{k=0}^{\infty}$. When $\alpha=0$ and $\beta=1$ we drop the corresponding arguments in the expressions of the generalized Horadam numbers.

Generalized Horadam numbers, encompasses many special second order sequences as generalized $F i$ bonacci, Lucas, Pell, Jacobsthal and Jacobsthal-Lucas numbers, that appear for specific values of the parameters $\tau, \xi, \alpha$ and $\beta$, see for instance $[2,19]$. The properties and relations of these sequences have been extensively treated elsewhere, see for instance $[2,12,19]$, and they are not the objective of this work. Here, we pay attention on the special kind of self-adjoint equations with constant coefficients, usually not considered between the above sequences. In this case, $a=c$, and hence the Equation (10) is equivalent to

$$
z(k+1)-2 q z(k)+z(k-1)=0, \quad k \in \mathbb{N}^{*},
$$

where $q=\frac{b}{2 a}$, that is known as the Chebyshev equation with parameter $q$. Notice that this case corresponds to the Horadam sequences $\left\{H_{k}(2 q,-1 ; \alpha, \beta)\right\}_{k=0}^{\infty}$. Although in the framework of number sequences, Chebyshev equations have received only scarce attention, they have an ubiquitous presence in many areas of applied mathematics, through the associated Chebyshev polynomials. Quoting the well known claim, commonly attributed to P. Davis and G. Forsythe, Chebyshev polynomials are everywhere dense in numerical analysis the first lines of the book [16] assert [...] there is scarcely any area of numerical analysis where Chebyshev polynomials do not drop in like surprise visitors, and indeed there are now a number of subjects in which these polynomials take a significant position in modern developments, including orthogonal 
polynomials, polynomial approximation, numerical integration, and spectral methods for partial differential equations.

Our objective in this section is to compute the Green function of the Equation (10). We start our analysis with the usual technique based on the relationship of the solutions and the roots of the Characteristic Polynomial of the equation, that is defined as $Q(x)=a x^{2}-b x+c \in \mathbb{K}[x]$. Note that always $Q(0)=c \neq 0$ and hence if $Q(r)=0$, necessarily $r \in \mathbb{K}^{*}$.

Lemma 8.1. Given $\nu \in \mathbb{K}$, consider $\hat{\nu}=a^{-1} b-\nu$ and $z, w, u \in \ell(\mathbb{K})$ the sequences defined respectively as $z(k)=\nu^{k}, w(k)=\hat{\nu}^{k}$ and $u(k)=k \nu^{k}, k \in \mathbb{N}$. The following results are satisfied:

(i) $z \in \mathcal{S}$ iff $Q(\nu)=0$. In this case, $Q(\hat{\nu})=0$ and moreover $\{z, w\}$ is a basis of $\mathcal{S}$ iff $b \neq 2 a \nu$.

(ii) $u \in \mathcal{S}$ iff $Q(x)=a(x-\nu)^{2}$; that is iff $Q(\nu)=0$ and $b=2 a \nu$. In this case, $\{z, u\}$ is a basis of $\mathcal{S}$.

Proof. (i) For any $k \in \mathbb{N}^{*}$ we obtain that

$$
a z(k+1)-b z(k)+c z(k-1)=a \nu^{k+1}-b \nu^{k}+c \nu^{k-1}=Q(\nu) \nu^{k-1} .
$$

Therefore, if $Q(\nu)=0$, then $z$ is a solution of the equation and conversely, if $z$ is a solution, taking $k=1$ in the above identity, we obtain that $Q(\nu)=0$. Moreover, since $Q(\hat{\nu})=Q(\nu)$ we also have that $w \in \mathcal{S}$ and $\omega[z, w](0)=\hat{\nu}-\nu$. Therefore, $\{z, w\}$ is a basis of $\mathcal{S}$ iff $\hat{\nu} \neq \nu$; that is, iff $b \neq 2 a \nu$.

(ii) For any $k \in \mathbb{N}^{*}$ we have that

$$
a u(k+1)-b u(k)+c u(k-1)=k\left[a \nu^{k+1}-b \nu^{k}+c \nu^{k-1}\right]+\left(a \nu^{2}-c\right) \nu^{k-1}=k Q(\nu)+\left(a \nu^{2}-c\right) \nu^{k-1}
$$

and hence reasoning $u$ is a solution iff $k Q(\nu)+\left(a \nu^{2}-c\right) \nu^{k-1}=0$ for any $k \in \mathbb{N}^{*}$. Since for $k=1$ we have $a \nu^{2}-c=-Q(\nu)$, we obtain that $u$ is a solution of the equation iff $Q(\nu)\left(k-\nu^{k-1}\right)=0$ for any $k \in \mathbb{N}^{*}$. Since the equations $2=\nu$ and $3=\nu^{2}$ form an inconsistent system, the above equality is true for any $k \in \mathbb{N}^{*}$ iff $Q(\nu)=0$, which implies also that $c=a \nu^{2}$ and hence that $b=2 a \nu$. Equivalently, $Q(x)=a(x-\nu)^{2}$. Finally, since $\omega[z, u](0)=\nu \neq 0$, we conclude that $\{z, u\}$ is a basis of $\mathcal{S}$.

Proposition 8.2 (Binet Formula). If there exists $\mu \in \mathbb{K}$ such that $\mu^{2}=b^{2}-4 a c$, then the Green function for the Equation (10) is given by one of the following expressions:

(i) $g(k, s)=\mu^{-1}\left[\left(\frac{b+\mu}{2 a}\right)^{k-s}-\left(\frac{b-\mu}{2 a}\right)^{k-s}\right], k, s \in \mathbb{N}$ when $\mu \neq 0$.

(ii) $g(k, s)=a^{-1}(k-s)\left(\frac{b}{2 a}\right)^{k-s-1}, k, s \in \mathbb{N}$, when $\mu=0$.

Corollary 8.3. The Equation (10) is exact iff $b=a+c$ and then its Green function is given by one of the following expressions:

(i) $g(k, s)=\frac{1}{a-c}\left[1-\left(\frac{c}{a}\right)^{k-s}\right], k, s \in \mathbb{N}$ when $a \neq c$.

(ii) $g(k, s)=a^{-1}(k-s), k, s \in \mathbb{N}$, when $a=c$.

Notice that according Corollary 6.4, the Green function for (10) when $b=a+c$ is given by

$$
g(k, s)=a^{-1}\left(\frac{a}{c}\right)^{s} \int_{s}^{k}\left(\frac{c}{a}\right)^{r-1} d r=a^{-1}\left(\frac{a}{c}\right)^{s} \sum_{r=s+1}^{k}\left(\frac{c}{a}\right)^{r-1}, \text { for any } k, s \in \mathbb{N},
$$

that clearly coincides with the identities in the above Corollary. 
The Binet Formula determines a simple procedure to obtain the Green function. Therefore, it applies to any equation equivalent to (10). The following result characterize when this happens.

Proposition 8.4. Consider $\hat{a}, \hat{c} \in \ell\left(\mathbb{K}^{*}\right), \hat{b} \in \ell(\mathbb{K})$, the difference equation

$$
\hat{a}(k) z(k+1)-\hat{b}(k) z(k)+\hat{c}(k-1) z(k-1)=0, \quad k \in \mathbb{N}^{*}
$$

and $\hat{\mathcal{S}}$ the space of its solutions. Then, the equation is equivalent to an equation with constant coefficients iff $\hat{\mathcal{S}}$ is shift-invariant and then it is equivalent to the equation

$$
\hat{a}(1) z(k+1)-\hat{b}(1) z(k)+\hat{c}(0) z(k-1)=0, \quad k \in \mathbb{N}^{*} .
$$

Proof. From Theorem 5.2, the equation is equivalent to another one with constant coefficients, say $a, c \in$ $\mathbb{K}^{*}$ and $b \in \mathbb{K}$ iff there exists $\gamma \in \ell\left(\mathbb{K}^{*}\right)$ such that $\hat{a}=\gamma a, \hat{b}=\gamma b$ and $\hat{c}=\gamma_{1} c$, or equivalently iff it is equivalent to the equation with coefficients $\hat{a}(1), \hat{b}(1)$ and $\hat{c}(0)$, since $\hat{a}(1)=\gamma(1) a, \hat{b}(1)=\gamma(1) b$ and $\hat{c}(0)=\gamma(1) c$.

Moreover, if $z \in \hat{\mathcal{S}}$, then satisfies the above equality for any $k \in \mathbb{N}^{*}$ and hence also satisfies it for any $k+1, k \in \mathbb{N}^{*}$; that is

$$
0=\hat{a}(1) z(k+2)-\hat{b}(1) z(k+1)+\hat{c}(0) z(k)=\hat{a}(1) z_{1}(k+1)-\hat{b}(1) z_{1}(k)+\hat{c}(0) z_{1}(k-1), \quad \text { for any } k \in \mathbb{N}^{*} .
$$

In other words, $z_{1}$ is also a solution; that is $\widehat{\mathcal{S}}$ is shift-invariant. Conversely, if we assume that $\hat{\mathcal{S}}$ is shift-invariant and consider $z \in \hat{S}$, for any $k \in \mathbb{N}^{*}$ we have

$$
\begin{aligned}
& z_{1}(k+1)=z(k+2)=\frac{\hat{b}(k+1)}{\hat{a}(k+1)} z(k+1)-\frac{\hat{c}(k)}{\hat{a}(k+1)} z(k) \\
& z_{1}(k+1)=\frac{\hat{b}(k)}{\hat{a}(k)} z_{1}(k)-\frac{\hat{c}(k-1)}{\hat{a}(k)} z_{1}(k-1)=\frac{\hat{b}(k)}{\hat{a}(k)} z(k+1)-\frac{\hat{c}(k-1)}{\hat{a}(k)} z(k)
\end{aligned}
$$

and subtracting both identities, we have

$$
0=z(k+1)\left(\frac{\hat{b}(k+1)}{\hat{a}(k+1)}-\frac{\hat{b}(k)}{\hat{a}(k)}\right)+z(k)\left(\frac{\hat{c}(k-1)}{\hat{a}(k)}-\frac{\hat{c}(k)}{\hat{a}(k+1)}\right), \quad k \in \mathbb{N}^{*} .
$$

Fixed $m \in \mathbb{N}^{*}$, if we consider the unique $z \in \hat{S}$ such that $z(m)=1$ and $z(m+1)=0$, then the above identity implies that $\frac{\hat{c}(m-1)}{\hat{a}(m)}=\frac{\hat{c}(m)}{\hat{a}(m+1)}$, whereas if we consider the unique $z \in \hat{S}$ such that $z(m)=0$ and $z(m+1)=1$, then $\frac{\hat{b}(m)}{\hat{a}(m)}=\frac{\hat{b}(m+1)}{\hat{a}(m+1)}$. Therefore, $a_{1}^{-1} c$ is constant and $a^{-1} b$ is constant in $\mathbb{N}^{*}$. Since the values $a(0)$ and $b(0)$ do not have relevance, we can assume that $a^{-1} b$ is constant in $\mathbb{N}$.

Notice that the reiteration of the above result establishes that if $z \in \mathcal{S}$, then $z_{s} \in \mathcal{S}$ for any $s \in \mathbb{N}$. This simple property generates some well known identities involving the sequences in $\mathcal{S}$, see [2, Formula (2.5)] or [19, Theorems 5 and 6$]$.

Proposition 8.5. Given $z \in \mathcal{S}$, then for any $s \in \mathbb{N}^{*}$ we have

$$
z(k+1) z(k+s)-z(k) z(k+s+1)=\left(\frac{c}{a}\right)^{k}(z(1) z(s)-z(0) z(s+1)), \quad k \in \mathbb{N}
$$


or, equivalently

$a z(k+1) z(k+s)-b z(k) z(k+s)+c z(k) z(k+s-1)=\left(\frac{c}{a}\right)^{k}(a z(1) z(s)-b z(0) z(s)+c z(0) z(s-1)), \quad k \in \mathbb{N}$.

In particular,

$$
z(k+1)^{2}-z(k) z(k+2)=\left(\frac{c}{a}\right)^{k}\left(z(1)^{2}-z(0) z(2)\right), \quad k \in \mathbb{N}
$$

or, equivalently

$$
a z(k+1)^{2}-b z(k) z(k+1)+c z(k)^{2}=\left(\frac{c}{a}\right)^{k}\left(a z(1)^{2}-b z(0) z(1)+c z(0)^{2}\right), \quad k \in \mathbb{N} .
$$

Proof. Bearing in mind that $z_{s} \in \mathcal{S}$ for any $s \in \mathbb{N}$, the first identity is a direct consequence of being $a \rho \omega\left[z, z_{s}\right]$ a constant function. The second identity follows applying that $z(k+s+1)=a^{-1}(b z(k+s)-$ $c z(k+s-1))$ for any $k \in \mathbb{N}^{*}$.

To obtain the Green function of a second order difference equation with constant coefficients from the Binet Formula we need the characteristic polynomial be reducible in $\mathbb{K}[x]$. So, to obtain the generalized Horadam numbers with this method, it is necessary that $\tau^{2}+4 \xi \geq 0$, that is the hypothesis in [19]. Otherwise, we can solve the equation on the algebraic closure of $\mathbb{K}$ or even in the quadratic extension $\mathbb{K}\left(\sqrt{b^{2}-4 a c}\right)$, or $\mathbb{K}\left(\sqrt{\tau^{2}+4 \xi}\right)$ for Horadam numbers, see [2].

In this paper, we follow a route different to the usual to find the Green function, since we use the results of previous sections that do not need any supplementary hypothesis on the coefficients. To do this, we adapt to this scenario the results about Chebyshev functions.

For any $k \in \mathbb{Z}, k \geq-1$, we define the $k$-th Chebyshev polynomial of second kind in two variables, say $x, y$, as the polynomial $u_{k}(x, y)=P_{k}(x, y) \in \mathbb{Z}[x, y] \subset \mathbb{K}[x, y]$, where $P_{k}$ is the $k$-th Chebyshev function on $\ell(\mathbb{K})$ and $x, y$ are identified with constant sequences. Therefore, $u_{0}(x, y)=1$ and $u_{-1}(x, y)=0$ and moreover, $\operatorname{since} \operatorname{card}\left(\ell_{k}^{m}\right)=\left(\begin{array}{c}k-m \\ m\end{array}\right)$ for any $k \in \mathbb{N}^{*}$, we obtain that

$$
u_{k}(x, y)=\sum_{m=0}^{\left\lfloor\frac{k}{2}\right\rfloor}(-1)^{m}\left(\begin{array}{c}
k-m \\
m
\end{array}\right) x^{k-2 m} y^{m} .
$$

Clearly, $u_{1}(x, y)=x, u_{2}(x, y)=x^{2}-y$ and $u_{k}(x, 0)=x^{k}, u_{2 k-1}(0, y)=0$ and $u_{2 k}(0, y)=(-1)^{k} y^{k}$ for any $k \in \mathbb{N}$. On the other hand, for any $k \geq-1$ we have

$$
U_{k}(x)=u_{k}(2 x, 1)=\sum_{m=0}^{\left\lfloor\frac{k}{2}\right\rfloor}(-1)^{m}\left(\begin{array}{c}
k-m \\
m
\end{array}\right)(2 x)^{k-2 m},
$$

that is known as the standard (one variable) $k$-th Chebyshev polynomial of second kind, see [2] and also $[5,7]$. This is the reason to name Chebyshev functions and polynomials to $P_{k}$ and $u_{k}, k \in \mathbb{N}$. Since $U_{-1}(x)=0$, the equality is also true for $k=-1$. In fact, we can extend the definition of Chebyshev polynomials to negative integer indexes. Specifically, when $y \in \mathbb{K}^{*}$, we define

$$
u_{-k}(x, y)=-y^{1-k} u_{k-2}(x, y), \quad k \in \mathbb{N} .
$$


Note that for for $k=0$ or $k=2$ the above identity simply says that $u_{-2}(x, y)=-y^{-1}=P_{-2}\left(x_{1}, y_{1}\right)$, whereas for $k=1$, we recover the definition of $u_{-1}$, since from (11), $u_{-1}(x, y)=0$.

As a by-product of the above identity, we have the following version of Lemma 7.2.

Lemma 8.6. Given $x \in \mathbb{K}, y, z \in \mathbb{K}^{*}$, then $u_{k}(z x, z y)=z^{k} u_{k}\left(x, z^{-1} y\right), k \in \mathbb{Z}$. In particular,

$$
u_{k}\left(2 x, y^{2}\right)=y^{k} U_{k}\left(y^{-1} x\right), \quad k \in \mathbb{Z}
$$

and hence, $u_{k}\left(2 x, x^{2}\right)=x^{k} U_{k}(1)=(k+1) x^{k}$.

Proposition 8.7. The Chebyshev polynomials of second kind in two variables are characterized by the three-terms recurrence

$$
u_{n+1}(x, y)=x u_{n}(x, y)-y u_{n-1}(x, y), \quad n \in \mathbb{N}, \quad u_{-1}(x, y)=0, \quad u_{0}(x, y)=1 .
$$

More generally, for any $r \geq-1$,

$$
u_{r}(x, y) u_{n+r+1}(x, y)=u_{r+1}(x, y) u_{n+r}(x, y)-y^{r+1} u_{n-1}(x, y), \quad n \in \mathbb{N}
$$

and when $y \in \mathbb{K}^{*}$, then the recurrences also are satisfied for $n, r \in \mathbb{Z}$.

Proof. For $n \in \mathbb{N}$, the first recurrence relation is a straightforward consequence of Proposition 7.3. To prove the second claim, for any $n \in \mathbb{N}$ and any $r \geq-1$ we define

$$
W_{n, r}(x, y)=u_{r+1}(x, y) u_{n+r}(x, y)-u_{r}(x, y) u_{n+r+1}(x, y)
$$

and then we have to prove that $W_{n, r}(x, y)=y^{r+1} u_{n-1}(x, y)$. Clearly, $W_{n,-1}(x, y)=u_{n-1}(x, y)$, whereas

$$
W_{n, 0}(x, y)=u_{1}(x, y) u_{n}(x, y)-u_{n+1}(x, y)=x u_{n}(x, y)-u_{n+1}(x, y)=y u_{n-1}(x, y),
$$

where we have used the above three terms recurrence. If $r>0$, then

$$
\begin{aligned}
W_{n, r}(x, y) & =\left[x u_{r}(x, y)-y u_{r-1}(x, y)\right] u_{n+r}(x, y)-u_{r}(x, y)\left[x u_{n+r}(x, y)-y u_{n+r-1}(x, y)\right] \\
& =y\left[u_{r}(x, y) u_{n+r-1}(x, y)-u_{r-1}(x, y) u_{n+r}(x, y)\right]=y W_{n, r-1}(x, y)
\end{aligned}
$$

and hence, $W_{n, r}(x, y)=y^{r} W_{n, 0}(x, y)=y^{r+1} u_{n-1}(x, y)$.

When $y \in \mathbb{K}^{*}$, then for $n=-1$, the right side of the first equality reads as $x u_{-1}(x, y)-y u_{-2}(x, y)=1$ that coincides with $u_{0}(x, y)$. When $n=-k$ where $k \geq 2$, applying the three-terms recurrence for non negative integers, we obtain that

$$
\begin{aligned}
x u_{-k}(x, y)-y u_{-(k+1)}(x, y) & =-x y^{1-k} u_{k-2}(x, y)+y^{1-k} u_{k-1}(x, y)=y^{1-k}\left[u_{k-1}(x, y)-x u_{k-2}(x, y)\right] \\
& =-y^{2-k} u_{k-3}(x, y)=u_{-k+1}(x, y),
\end{aligned}
$$

which implies that first claimed recurrence also holds for negative integers.

Finally, considering $W_{n, r}(x, y)$ for $n, r \in \mathbb{Z}$, we easily obtain that $W_{n, r}(x, y)=y W_{n, r-1}(x, y)$. Therefore, when $r \geq 0$ we get that $W_{n, r}(x, y)=y^{r} W_{n, 0}(x, y)=y^{r+1} u_{n-1}(x, y)$. On the other hand, when $r=-k$ where $k>0$, then $W_{n, r}(x, y)=W_{n,-k}(x, y)=y^{-1} W_{n, 1-k}(x, y)$ and hence $W_{n, r}(x, y)=y^{-k} W_{n, 0}(x, y)=$ $y^{r} W_{n, 0}(x, y)=y^{r+1} u_{n-1}(x, y)$. 
Choosing $n=1$ in the second recurrence, we obtain that

$$
u_{r+1}(x, y)^{2}-u_{r+2}(x, y) u_{r}(x, y)=y^{r+1}, \quad r \geq-1 .
$$

In addition, when $y \in \mathbb{K}^{*}$, this identity is also satisfied for any $r \in \mathbb{Z}$. Note that as a by-product we obtain the following well-known identities involving Chebyshev polynomials of second kind,

$$
U_{r+1}(x) U_{n+r}(x)-U_{r}(x) U_{n+r+1}(x)=U_{n-1}(x), \quad n, r \in \mathbb{Z}
$$

and, in particular, $U_{r+1}(x)^{2}-U_{r}(x) U_{r+2}(x)=1$, for any $r \in \mathbb{Z}$.

Proposition 8.8. The Green function for the Equation (10) is

$$
g(k, s)=a^{s-k} u_{k-s-1}(b, a c), \quad k, s \in \mathbb{N} .
$$

Proof. Taking into account that for any constant sequence any shift coincides with it, according with Theorem 7.4 we have

$$
g(k, s)=\left\{\begin{array}{cc}
a^{s-k} u_{k-s-1}(b, a c), & k \geq s \\
-c^{k-s} u_{s-k-1}(b, a c), & k \leq s
\end{array}\right.
$$

Moreover, since $a c \in \mathbb{K}^{*}$, the Identity (11) implies that when $k \leq s$

$$
u_{k-s-1}(b, a c)=u_{-(s+1-k)}(b, a c)=-(a c)^{k-s} u_{s-k-1}(b, a c)
$$

and the expression for the Green function follows.

Corollary 8.9. When Equation (10) is uncoupled; that is, when $b=0$, its Green function is given by

$$
\begin{array}{clrl}
g(2 k+1,2 s) & =(-1)^{k-s} a^{-1}\left(\frac{c}{a}\right)^{k-s}, & & k, s \in \mathbb{N}, \\
g(2 k, 2 s+1) & =(-1)^{k-s-1} c^{-1}\left(\frac{c}{a}\right)^{k-s}, & & k, s \in \mathbb{N}, \\
g(m, n) & =0, & & \text { otherwise. }
\end{array}
$$

The case of exact equations, leads to the following property of the Chebyshev polynomials.

Corollary 8.10. For any $x, y \in \mathbb{K}$ it is satisfied that

$$
\sum_{m=0}^{\left\lfloor\frac{k}{2}\right\rfloor}(-1)^{m}\left(\begin{array}{c}
k-m \\
m
\end{array}\right)(x+y)^{k-2 m}(x y)^{m}=\sum_{j=0}^{k} y^{k-j} x^{j}, \quad k \in \mathbb{N} .
$$

Notice that for $x \in \mathbb{K}^{*}$ and $y=x^{-1}$ the above identity becomes the well-known expression

$$
U_{k}\left(2^{-1}\left(x+x^{-1}\right)\right)=\frac{x^{k+1}-x^{-k-1}}{x-x^{-1}}, \quad k \in \mathbb{N},
$$

see for instance [16, Identity 1.51]. 
If $\phi(k)=g(k, 0)=a^{-k} u_{k-1}(b, a c), k \in \mathbb{N}$, then $\phi(0)=0, \phi(1)=a^{-1}$ and moreover we can evaluate $\phi$ at negative integers, since we have extend the definition of the Chebyshev polynomials to this kind of indexes. In fact, we could have extend the definition of the equation $a z(k+1)-b z(k)+c z(k-1)=0$ to any $k \in \mathbb{Z}$. Since $a c \in \mathbb{K}^{*}$, Proposition 8.7 shows that $g(\cdot, s)$ satisfies this equation for fixed $s \in \mathbb{Z}$. Therefore, $\phi$ is the unique solution of the above equation, $k \in \mathbb{Z}$, satisfying $\phi(0)=0$ and $\phi(1)=a^{-1}$ and is called the fundamental or the primary solution, see [2], because $g(k, s)=\phi(k-s)$ for any $k, s \in \mathbb{N}$. This is also a characteristic of second order difference equation with constant coefficients: their Green function depends only on the difference between its arguments.

The ubiquitous presence of Chebyshev polynomials and its relation with difference equations with constant coefficients is described in the following result, see [2, Theorem 1]. It represents an alternative to Binet Formula.

Corollary 8.11. Given $a, c \in \mathbb{K}^{*}$ and $b \in \mathbb{K}$, assume that there exists $\theta \in \mathbb{K}$ such that $\theta^{2}=a c$. Then the Green function for the homogeneous difference equation $a z(k+1)-b z(k)+c z(k-1)=0, k \in \mathbb{N}^{*}$, is

$$
g(k, s)=a^{-1}\left(a^{-1} \theta\right)^{k-s-1} U_{k-s-1}\left(\frac{b}{2 \theta}\right), \text { for any } k, s \in \mathbb{N} .
$$

Proof. Taking $x=\frac{b}{2}$, since $\theta \in \mathbb{K}^{*}$, applying the second part of Lemma 8.6, we obtain that,

$$
u_{k-s-1}(b, a c)=u_{k-s-1}\left(2 x, \theta^{2}\right)=\theta^{k-s-1} U_{k-s-1}\left(\theta^{-1} x\right)=\theta^{k-s-1} U_{k-s-1}\left((2 \theta)^{-1} b\right) .
$$

The result follows from the above Proposition.

As in the case of Binet Formula, if $x^{2}-a c$ is not reducible in $\mathbb{K}[x]$ the identity in the above Corollary has meaning in the algebraic closure of $\mathbb{K}$ or even in the quadratic extension $\mathbb{K}(\sqrt{a c})$. However, unlike Binet Formula, we can apply another property of the solutions of second order difference equations with constant coefficients to avoid using $\mathbb{K}(\sqrt{a c})$.

Lemma 8.12. Given $z \in \mathcal{S}$, for any $m \in \mathbb{N}$ the sequence $w \in \ell(\mathbb{K})$ defined as $w(k)=z(2 k+m)$ is a solution of the equation

$$
a^{2} w(k+1)-\left(b^{2}-2 a c\right) w(k)+c^{2} w(k-1)=0, \quad k \in \mathbb{N}^{*} .
$$

Proof. Since $z \in \mathcal{S}$, for any $k \in \mathbb{N}^{*}$ we have

$$
\begin{aligned}
a^{2} w(k+1) & =a^{2} z(2 k+m+2)=b a z(2 k+m+1)-a c z(2 k+m) \\
& =b[b z(2 k+m)-c z(2 k+m-1)]-a c z(2 k+m) \\
& =\left(b^{2}-a c\right) z(2 k+m)-c b z(2 k+m-1) \\
& =\left(b^{2}-a c\right) z(2 k+m)-c[a z(2 k+m)+c z(2 k+m-2)] \\
& =\left(b^{2}-2 a c\right) z(2 k+m)-c^{2} z(2 k+m-2)=\left(b^{2}-2 a c\right) w(k)-c^{2} w(k-1) .
\end{aligned}
$$

Proposition 8.13. Given $a, c \in \mathbb{K}^{*}$ and $b \in \mathbb{K}$, the Green function for the homogeneous difference equation 
$a z(k+1)-b z(k)+c z(k-1)=0, k \in \mathbb{N}^{*}$, is determined by

$$
\begin{aligned}
g(2 k, 2 s) & =g(2 k+1,2 s+1)=b a^{-2}\left(a^{-1} c\right)^{k-s-1} U_{k-s-1}\left(\frac{b^{2}}{2 a c}-1\right), & & k, s \in \mathbb{N}, \\
g(2 k+1,2 s) & =a^{-3}\left(a^{-1} c\right)^{k-s-1}\left[\left(b^{2}-a c\right) U_{k-s-1}\left(\frac{b^{2}}{2 a c}-1\right)-a c U_{k-s-2}\left(\frac{b^{2}}{2 a b}-1\right)\right] & & k, s \in \mathbb{N}, \\
g(2 k, 2 s+1) & =a^{-3}\left(a^{-1} c\right)^{k-s-2}\left[\left(b^{2}-a c\right) U_{k-s-2}\left(\frac{b^{2}}{2 a c}-1\right)-a c U_{k-s-3}\left(\frac{b^{2}}{2 a c}-1\right)\right], & & k, s \in \mathbb{N} .
\end{aligned}
$$

Proof. If $\phi$ is the primary solution of the equation, we know that $g(k, s)=\phi(k-s)$ for any $k, s \in \mathbb{N}$. Moreover, $\phi(0)=0$ and $\phi(1)=a^{-1}$ which implies that $\phi(2)=a^{-2} b$ and $\phi(3)=a^{-3}\left(b^{2}-a c\right)$

On the other hand, if we consider $u, v \in \ell(\mathbb{K})$ defined as $u(k)=\phi(2 k)$ and $v(k)=\phi(2 k+1), k \in \mathbb{N}$ the above Lemma assures that $u$ and $v$ are solutions of the equation

$$
a^{2} z(k+1)-\left(b^{2}-2 a c\right) z(k)+c^{2} z(k-1)=0, \quad k \in \mathbb{N}^{*} .
$$

Therefore, if $\tilde{g}$ is the Green function of the above equation, then from Proposition 4.3 we have

$$
\begin{aligned}
& u(k)=a^{2} u(1) \tilde{g}(k, 0)-c^{2} u(0) \tilde{g}(k, 1)=b \tilde{g}(k, 0), \\
& v(k)=a^{2} v(1) \tilde{g}(k, 0)-c^{2} v(0) \tilde{g}(k, 1)=a^{-1}\left(b^{2}-a c\right) \tilde{g}(k, 0)-a^{-1} c^{2} \tilde{g}(k, 1) .
\end{aligned}
$$

Since $\theta=a c$ is a root of the polinomial $x^{2}-a^{2} c^{2}$, from Corollary 8.11 we know that

$$
\tilde{g}(k, s)=a^{-2}\left(a^{-1} c\right)^{k-s-1} U_{k-s-1}\left(\frac{b^{2}}{2 a c}-1\right), \quad k, s \in \mathbb{N}
$$

and hence,

$$
\begin{aligned}
& u(k)=b a^{-2}\left(a^{-1} c\right)^{k-1} U_{k-1}\left(\frac{b^{2}}{2 a c}-1\right), \\
& v(k)=a^{-3}\left(a^{-1} c\right)^{k-1}\left[\left(b^{2}-a c\right) U_{k-1}\left(\frac{b^{2}}{2 a c}-1\right)-a c U_{k-2}\left(\frac{b^{2}}{2 a c}-1\right)\right] .
\end{aligned}
$$

Taking into account that $U_{k}(-x)=(-1)^{k} U_{k}(x)$ for any $k \in \mathbb{Z}$ and any $x \in \mathbb{C}$, we have the following expressions for the generalized Horadam numbers, see [6].

Corollary 8.14. Given $\tau, \xi \in \mathbb{R}$ and $\xi \neq 0$, we have the following results:

(i) If $\xi<0$, then $H_{k}(\tau, \xi)=(\sqrt{-\xi})^{k-1} U_{k-1}\left(\frac{\tau}{2 \sqrt{-\xi}}\right), k \in \mathbb{N}$.

(ii) If $\xi>0$, then $H_{k}(\tau, \xi)=(-i)^{k-1}(\sqrt{\xi})^{k-1} U_{k-1}\left(\frac{i \tau}{2 \sqrt{\xi}}\right), k \in \mathbb{N}$ and also

$$
\begin{aligned}
H_{2 k}(\tau, \xi) & =\tau \xi^{k-1} U_{k-1}\left(1+\frac{\tau^{2}}{2 \xi}\right), & & k \in \mathbb{N}, \\
H_{2 k+1}(\tau, \xi) & =\xi^{k-1}\left[\left(\tau^{2}+\xi\right) U_{k-1}\left(1+\frac{\tau^{2}}{2 \xi}\right)-\xi U_{k-2}\left(1+\frac{\tau^{2}}{2 \xi}\right)\right], & & k \in \mathbb{N} .
\end{aligned}
$$

In the Lemma 8.12 we have directly proved a result that, in fact, represents an specialization of a deeper property of solutions of second order homogeneous difference equation with constant coefficients. To show this property, we need to introduce another kind of Chebyshev polynomials in two variables, see [7] for a more complete motivation. Prior to do this, we introduce the following notation: Given $P(x, y) \in \mathbb{K}[x, y]$ 
such that

$$
P(x, y)=\sum_{j=0}^{n} R_{j}(y) x^{j}, \quad \text { where } R_{j} \in \mathbb{K}[y], j=0, \ldots, n,
$$

then $P^{\prime}$ denotes the polynomial

$$
P^{\prime}(x, y)=\sum_{j=1}^{n} j R_{j}(y) x^{j-1}=\sum_{j=0}^{n-1}(j+1) R_{j+1}(y) x^{j} .
$$

In particular, if $P(x)=\sum_{j=0}^{n} r_{j} x^{j} \in \mathbb{K}[x]$, where $r_{j} \in \mathbb{K}, j=0, \ldots, n$ then $P^{\prime}(x)=\sum_{j=0}^{n-1}(j+1) r_{j+1} x^{j}$.

Lemma 8.15. If $P \in \mathbb{K}[x]$ satisfies that $P(b)=0$ for $b \in \mathbb{K}$, then $P(x)=R(x)(x-b)$ where $R \in \mathbb{K}[x]$ and moreover $R(b)=P^{\prime}(b)$.

The $k$-th Chebyshev polynomial of first kind in two variables $x, y$, is the polynomial $t_{k}(x, y) \in \mathbb{Z}[x, y] \subset$ $\mathbb{K}[x, y]$, defined as $t_{0}(x, y)=2$ and for any $k \in \mathbb{N}^{*}$ as

$$
t_{k}(x, y)=k \sum_{m=0}^{\left\lfloor\frac{k}{2}\right\rfloor} \frac{(-1)^{m}}{k-m}\left(\begin{array}{c}
k-m \\
m
\end{array}\right) x^{k-2 m} y^{m}
$$

Clearly, $t_{1}(x, y)=x, t_{2}(x, y)=x^{2}-2 y$ and moreover $t_{k}(x, 0)=x^{k}, t_{2 k-1}(0, y)=0$ and $t_{2 k}(0, y)=2(-1)^{k} y^{k}$ for any $k \in \mathbb{N}$. On the other hand, for any $k \in \mathbb{N}$ we have

$$
T_{k}(x)=2^{-1} t_{k}(2 x, 1)=\frac{k}{2} \sum_{m=0}^{\left\lfloor\frac{k}{2}\right\rfloor} \frac{(-1)^{m}}{k-m}\left(\begin{array}{c}
k-m \\
m
\end{array}\right)(2 x)^{k-2 m},
$$

that is known as the standard (one variable) $k$-th Chebyshev polynomial of first kind, see [2] and also [5, 7]. Notice that when $y \neq 0$,

$$
t_{k}\left(2 x, y^{2}\right)=2 y^{k} T_{k}\left(y^{-1} x\right), \quad k \in \mathbb{N},
$$

and hence, $t_{k}\left(2 x, x^{2}\right)=2 x^{k} T_{k}(1)=2 x^{k}$. As in the case of Chebyshev polynomials of second kind, we can extend the definition of Chebyshev polynomials of first kind to negative integer indexes. Specifically, when $y \in \mathbb{K}^{*}$, we define

$$
t_{-k}(x, y)=y^{-k} t_{k}(x, y), \quad k \in \mathbb{N} .
$$

As in the standard setting, the two kind of Chebyshev polynomials in two variables are closely related. Next, we describe this relation and show a characterization of the first kind polynomials that in fact implies that they generate solutions of the Equation (10), see Corollary 8.17 below.

Proposition 8.16. For any $x, y \in \mathbb{K}$ we have that

$$
t_{k}(x, y)=u_{k}(x, y)-y u_{k-2}(x, y) \quad \text { and } \quad t_{k}^{\prime}(x, y)=k u_{k-1}(x, y), \quad k \in \mathbb{N} .
$$


In particular, the Chebyshev polynomials of first kind in two variables are characterized by the threeterms recurrence

$$
t_{k+2}(x, y)=x t_{k+1}(x, y)-y t_{k}(x, y), \quad k \in \mathbb{N}^{*}, \quad t_{0}(x, y)=2, \quad t_{1}(x, y)=x
$$

Moreover if $y \in \mathbb{K}^{*}$ then all the identities are also satisfied for $k \in \mathbb{Z}$.

Proof. Since $u_{-2}(x, y)=-y^{-1}, u_{-1}(x, y)=0$ and $t_{0}^{\prime}(x, y)=0$, the first identity is true for $k=0,1$ and the second one is true for $k=0$. On the other hand, bearing in mind that $\left\lfloor\frac{k+1}{2}\right\rfloor=\left\lfloor\frac{k-1}{2}\right\rfloor+1$, when $k \in \mathbb{N}^{*}$ we have

$$
\begin{aligned}
u_{k+1}(x, y)-y u_{k-1}(x, y) & =\sum_{m=0}^{\left\lfloor\frac{k+1}{2}\right\rfloor}(-1)^{m}\left(\begin{array}{c}
k+1-m \\
m
\end{array}\right) x^{k+1-2 m} y^{m}-y \sum_{m=0}^{\left\lfloor\frac{k-1}{2}\right\rfloor}(-1)^{m}\left(\begin{array}{c}
k-1-m \\
m
\end{array}\right) x^{k-1-2 m} y^{m} \\
& =\sum_{m=0}^{\left\lfloor\frac{k+1}{2}\right\rfloor}(-1)^{m}\left(\begin{array}{c}
k+1-m \\
m
\end{array}\right) x^{k+1-2 m} y^{m}+\sum_{m=1}^{\left\lfloor\frac{k-1}{2}\right\rfloor+1}(-1)^{m}\left(\begin{array}{c}
k-m \\
m-1
\end{array}\right) x^{k+1-2 m} y^{m} \\
& =x^{k+1}+\sum_{m=1}^{\left\lfloor\frac{k+1}{2}\right\rfloor}(-1)^{m}\left[\left(\begin{array}{c}
k+1-m \\
m
\end{array}\right)+\left(\begin{array}{c}
k-m \\
m-1
\end{array}\right)\right] x^{k+1-2 m} y^{m} \\
& =\frac{(k+1)}{k+1} x^{k+1}+\sum_{m=1}^{\left\lfloor\frac{k+1}{2}\right\rfloor}(-1)^{m} \frac{(k+1)}{k+1-m}\left(\begin{array}{c}
k+1-m \\
m
\end{array}\right) x^{k+1-2 m} y^{m}=t_{k+1}(x, y),
\end{aligned}
$$

and also that

$$
\begin{aligned}
t_{k}^{\prime}(x, y) & =k \sum_{m=0}^{\left\lfloor\frac{k-1}{2}\right\rfloor} \frac{(-1)^{m}(k-2 m)}{k-m}\left(\begin{array}{c}
k-m \\
m
\end{array}\right) x^{k-2 m-1} y^{m}=k \sum_{m=0}^{\left\lfloor\frac{k-1}{2}\right\rfloor}(-1)^{m}\left(\begin{array}{c}
k-1-m \\
m
\end{array}\right) x^{k-2 m-1} y^{m} \\
& =k u_{k-1}(x, y) .
\end{aligned}
$$

When $y \in \mathbb{K}^{*}$, then for any $k \in \mathbb{N}^{*}$ and taking into account that $u_{-k}(x, y)=-y^{1-k} u_{k-2}(x, y)$, we have

$$
\begin{aligned}
t_{-k}(x, y) & =y^{-k} t_{k}(x, y)=y^{-k}\left[u_{k}(x, y)-y u_{k-2}(x, y)\right]=y^{-k}\left[-y^{k+1} u_{-k-2}+y y^{k-1} u_{-k}(x, y)\right] \\
& =u_{-k}(x, y)-y u_{-k-2} \\
t_{-k}^{\prime}(x, y) & =y^{-k} t_{k}^{\prime}(x, y)=k y^{-k} u_{k-1}(x, y)=-k y^{-k} y^{k} u_{-k-1}=-k u_{-k-1}
\end{aligned}
$$

and hence the identities are also true for negative indexes.

Finally, the three-term characterization, and its extension to negative indexes when $y \in \mathbb{K}^{*}$, appears then as a consequence of the Proposition 8.7 together with the first identity.

Corollary 8.17. If $z \in \ell(\mathbb{K})$ is defined as $z(k)=a^{-k} t_{k}(b, a c), k \in \mathbb{Z}$, then $z \in \mathcal{S}$.

We are ready to show the mentioned generalization of Lemma 8.12 that is based in that constant sequences are periodic sequences with period $p$ for any $p \in \mathbb{N}$.

Theorem 8.18. Given $z \in \mathcal{S}$, for any $p, m \in \mathbb{N}$ the sequence $w \in \ell(\mathbb{K})$ defined as $w(k)=z(p k+m)$ is a 
solution of the equation

$$
a^{p} w(k+1)-t_{p}(b, a c) w(k)+c^{p} w(k-1)=0, \quad k \in \mathbb{N}^{*} .
$$

Proof. Consider $\theta=\sqrt{a c}$ a solution of the equation $x^{2}=a c$ in the quadratic extension $\mathbb{K}(\sqrt{a c})$. Then $\gamma=a^{-1} \theta \in \mathbb{K}(\sqrt{a c})$ and hence from [7, Theorem 2.4], see also [2, Theorem 3.1], $u(k)=\gamma^{-k} z(k)$, where $u$ is a solution of the Chebyshev equation with parameter $q=\frac{b}{2 \theta}$.

From [7, Corollary 4.6], we know that for any $p, m \in \mathbb{N}$ the sequence defined as $v(k)=u(p k+m)$ is a solution of the Chebyshev equation with parameter $T_{p}(q)$; which implies that

$$
0=v(k+1)-2 T_{p}(q) v(k)+v(k-1)=\gamma^{-(p k+m)}\left[\gamma^{-p} w(k+1)-2 T_{p}(q) w(k)+\gamma^{p} w(k-1)\right], \quad k \in \mathbb{N}^{*} .
$$

From the identity (12) we obtain that

$$
2 T_{p}(q)=\theta^{-p} t_{p}\left(b, \theta^{2}\right)=a^{-p} \gamma^{-p} t_{p}(b, a c), \quad p \in \mathbb{N}^{*}
$$

and hence,

$$
0=\gamma^{-p} w(k+1)-a^{-p} \gamma^{-p} t_{p}(b, a c) w(k)+\gamma^{p} w(k-1), \quad k \in \mathbb{N},
$$

or equivalently,

$$
0=a^{p} w(k+1)-t_{p}(b, a c) w(k)+a^{p} \gamma^{2 p} w(k-1), \quad k \in \mathbb{N},
$$

and the result follows from the equality $a^{p} \gamma^{2 p}=a^{p} a^{-2 p}(a c)^{p}=c^{p}$.

The reiteration of the above result, leads to the following property about the composition of Chebyshev polynomials of first kind.

Corollary 8.19. For any $n, m \in \mathbb{N}$ we have that

$$
t_{m}\left(t_{n}(x, y), y^{n}\right)=t_{m n}(x, y)
$$

and, in particular $T_{m n}(x)=T_{m}\left(T_{n}(x)\right)$.

Proof. It suffices to take into account that $t_{k}(2 x, 1)=2 T_{k}(x)$ for any $k \in \mathbb{N}$.

As a nice by-product of the above Theorem we have the following characterization of the existence of periodic solutions, see [7, Corollary 4.8] for the general case. Previously, associated with Equation (10) we define the sequence $\kappa \in \ell(\mathbb{K})$ as

$$
\kappa(k)=a^{k}+c^{k}-t_{k}(b, a c)=a^{k}+c^{k}-k \sum_{m=0}^{\left\lfloor\frac{k}{2}\right\rfloor} \frac{(-1)^{m}}{k-m}\left(\begin{array}{c}
k-m \\
m
\end{array}\right) b^{k-2 m}(a c)^{m}, \quad k \in \mathbb{N} .
$$

Notice that $\kappa(0)=0, \kappa(1)=a+c-b$ and $\kappa(2)=(a+c)^{2}-b^{2}$.

Corollary 8.20. The Equation (10) has non-null periodic solutions with period $p \in \mathbb{N}^{*}$ iff $\kappa(p)=0$

Proof. It suffices to observe that if $z \in \mathcal{S}$, then $z$ is periodic with period $p \in \mathbb{N}$ iff for any $0 \leq m<p$ the sequence $w \in \ell(\mathbb{K})$ given by $w(k)=z(p k+m), k \in \mathbb{N}$, is constant and then, apply the above Theorem. 
We remark that when $\kappa(p)=0, p \geq 2$, to obtain a periodic solution for the equation (10) it suffices to consider $\alpha, \beta \in \mathbb{K}$,

$$
z(k)=a^{1-k}\left[\beta u_{k-1}(b, a c)-c \alpha u_{k-2}(b, a c)\right], \quad k=0, \ldots, p-1
$$

and then define $z(p k+m)=z(m)$ for any $k \in \mathbb{N}^{*}$ and $0 \leq m<p-1$. Notice that the choice $\alpha=\beta$ determines a periodic solution which two first value are equal. Clearly this happens when $p=1$, since then the constant sequences are solution of (10).

The above corollary characterizes the existence of periodic solutions of the Equation (10). Since periodic sequences of period $p$ are periodic with period $m p$ for any $m \in \mathbb{N}^{*}$ the identity $a^{p}+c^{p}=t_{p}(b, a c)$ also implies that $a^{m p}+c^{m p}=t_{m p}(b, a c)$ for any $m \in \mathbb{N}^{*}$. In particular, the case $p=1$ reads that $a^{m}+c^{m}=t_{m}(b, a c)$.

Corollary 8.21. For any $x, y \in \mathbb{K}$ it is satisfied that

$$
k \sum_{m=0}^{\left\lfloor\frac{k}{2}\right\rfloor} \frac{(-1)^{m}}{k-m}\left(\begin{array}{c}
k-m \\
m
\end{array}\right)(x+y)^{k-2 m}(x y)^{m}=x^{k}+y^{k}, \quad k \in \mathbb{N} .
$$

Notice that for $x \in \mathbb{K}^{*}$ and $y=x^{-1}$ the above identity becomes the well-known expression

$$
T_{k}\left(2^{-1}\left(x+x^{-1}\right)\right)=\frac{x^{k}+x^{-k}}{2}, \quad k \in \mathbb{N},
$$

see for instance [16, Identity 1.47].

When $a=c$, then (10) is equivalent to a Chebyshev equation with parameter $q=\frac{b}{2 a}$. Then the above results establishes that the equation has non-null periodic solutions with period $n \in \mathbb{N}^{*}$ iff

$$
2 a^{n}=t_{n}\left(b, a^{2}\right)=2 a^{n} T_{n}(q)
$$

and hence, iff $T_{n}(q)=1$. When $\mathbb{K}=\mathbb{R}$, this happens iff $q=\cos \left(\frac{2 j \pi}{n}\right), j=0, \ldots,\left\lceil\frac{n-1}{2}\right\rceil$.

We end this work applying the results of Propositions 5.4 and 6.5 to the case of equations with constant coefficients. To do this, remember that for any $x \in \mathbb{K}$ all the solutions of the homogeneous equation

$$
c w(k+1)-x w(k)+a w(k+1)=0, \quad k \in \mathbb{N}^{*}
$$

can be written as

$$
w(x ; k)=c^{-k}\left[\alpha u_{k-1}(x, a c)+\beta u_{k-2}(x, a c)\right], \quad k \in \mathbb{N},
$$

where $\alpha, \beta \in \mathbb{K}$. Then, we can consider $w(x ; k)$ as a polynomial and hence it has sense $w^{\prime}(x ; k)$.

Proposition 8.22. Consider $\alpha, \beta, x \in \mathbb{K}$ and $w(x ; k)=c^{-k}\left[\alpha u_{k-1}(x, a c)+\beta u_{k-2}(x, a c)\right], k \in \mathbb{N}$. Then, for any $z \in \mathcal{S}$ and any $m, n \in \mathbb{N}$ such that $m \leq n$ we have

$\int_{m}^{n} z(s) w(x ; s) \nabla s=\frac{1}{x-b}[a(z(m+1) w(x ; m)-z(n+1) w(x ; n))+c(z(n) w(x ; n+1)-z(m) w(x ; m+1))]$, 
when $x \neq b$, whereas

$$
\int_{m}^{n} z(s) w(b ; s) \nabla s=a\left(z(m+1) w^{\prime}(b ; m)-z(n+1) w^{\prime}(b ; n)\right)+c\left(z(n) w^{\prime}(b ; n+1)-z(m) w^{\prime}(b ; m+1)\right) .
$$

In particular, when $a=c$ if we consider $\alpha=a z(1)$ and $\beta=-a^{2} z(0)$, then $w(b ; k)=z(k)$ and hence

$$
\int_{m}^{n} z(s)^{2} \nabla s=a\left[z(m+1) z^{\prime}(b ; m)-z(n+1) z^{\prime}(b ; n)+z(n) z^{\prime}(b ; n+1)-z(m) z^{\prime}(b ; m+1)\right] .
$$

Given $p \in \mathbb{N}$, if $x=a^{p}+c^{p}$, the equation $c^{p} w(k+1)-x w(k)+a^{p} w(k+1)=0, k \in \mathbb{N}^{*}$ is exact and hence from Corollary 8.3, \{1, $\zeta\}$ is a basis of the space of its solutions, where for any $k \in \mathbb{N}, \zeta(k)=\left(\frac{a}{c}\right)^{p k}$ when $a^{p} \neq c^{p}$ whereas $\zeta(k)=k$ when $a^{p}=c^{p}$. Then, the above Proposition together Theorem 8.18 provides the following summation formulas, see [19, Theorem 4].

Corollary 8.23. Consider $n, m, p, k \in \mathbb{N}$ such that $m \leq n$ and $\kappa(p) \neq 0$. Then, for any $z \in \mathcal{S}$ we have that

$$
\int_{m}^{n} z(p s+k) \nabla s=\frac{1}{\kappa(p)}\left[a^{p}(z(p(m+1)+k)-z(p(n+1)+k))+c^{p}(z(p n+k)-z(p m+k))\right] .
$$

In addition, when $a^{p} \neq c^{p}$, then

$$
\begin{aligned}
\int_{m}^{n}\left(\frac{a}{c}\right)^{p s} z(p s+k) \nabla s & =\frac{a^{p}}{\kappa(p)}\left[z(p(m+1)+k)\left(\frac{a}{c}\right)^{p m}-z(p(n+1)+k)\left(\frac{a}{c}\right)^{p n}\right] \\
& +\frac{c^{p}}{\kappa(p)}\left[z(p n+k)\left(\frac{a}{c}\right)^{p(n+1)}-z(p m+k)\left(\frac{a}{c}\right)^{p(m+1)}\right],
\end{aligned}
$$

whereas if $a=c$, then

$$
\int_{m}^{n} s z(p s+k) \nabla s=\frac{a^{p}}{\kappa(p)}[m z(p(m+1)+k)-n z(p(n+1)+k)+(n+1) z(p n+k)-(m+1) z(p m+k)] .
$$

\section{Funding}

This work has been partly supported by the Spanish Program I+D+i (Ministerio de Economía y Competitividad) under project MTM2014-60450-R.

\section{References}

[1] R.P. Agarwal Difference Equations and Inequalities, Marcel Dekker, 2000.

[2] D. Aharanov, A. Beardon, and K. Driver, Fibonacci, Chebyshev and orthogonal polynomials, Am. Math. Mon. 112 (2005), pp. 612-630.

[3] A. Avila and S. Jitomirskaya The Ten Martini problem, Ann. Math. (2) 170 (2009), pp. 303-342.

[4] H. Belbachir, T. Komatsu, and L. Szalay Linear recurrences associated to rays in Pascal's triangle and combinatorial identities. Math. Slovaca 64 (2014), pp. 287-300.

[5] E. Bendito, A. Carmona, and A.M. Encinas Eigenvalues, eigenfunctions and Green functions on a path via Chebyshev polynomials, Appl. Anal. Discret. Math. 3 (2009), pp. 282-302. 
[6] A. Carmona, A. M. Encinas, S. Gago, M.J. Jiménez, and M. Mitjana The inverses of some circulant matrices. Appl. Math. Comput. 270 (2015), pp. 785-793.

[7] A.M. Encinas and M.J. Jiménez, Floquet theory for second order linear homogeneous difference equations, J. Differ. Equ. Appl. 22 (2016), pp. 353-375.

[8] A.M. Encinas and M.J. Jiménez, Bounded solutions of self-adjoint second order linear difference equations with periodic coefficients, submitted.

[9] I. Gonoskov, Closed-form solution of a general three-term recurrence relation, Adv. Differ. Equ. 196 (2014), $12 \mathrm{pp}$.

[10] M.J. Jiménez and A.M. Encinas, Combinatorial Recurrences and Linear Difference Equations, Electron. Notes Discrete Math., 54 (2016), 313-318.

[11] I. Kaddoura and B. Mourad, On a new closed formula for the solution of homogeneous second order linear difference equations with constant coefficients and some applications, preprint (2017), to appear in J. Diff. Eq. Appl.

[12] T. Koshy, Fibonacci and Lucas Numbers with Applications, Wiley, New York, 2001.

[13] L. Liu and Y. Wang, On the log-convexity of combinatorial sequences, Adv. Appl. Math. 39 (2007), pp. 453-476.

[14] R.K. Mallik, On the solution of a second order linear homogeneous difference equation with variable coefficients, J. Math. Anal. Appl. 215 (1997), pp. 32-47.

[15] R.K. Mallik, Solutions of linear difference equations with variable coefficients, J. Math. Anal. Appl. 222 (1998), pp. $79-91$.

[16] J.C. Mason and D.C. Handscomb, Chebyshev Polynomials, Chapman \& Hall/CRC, 2003.

[17] E.D. Rainville, Special Functions, Chelsea Publishing Company, New York, 1960.

[18] G. Stolz and R. Weikard, Seminar on Jacobi matrices, Technical report, University of Alabama at Birmingham, 2004. Available at people.cas.uab.edu/ weikard/teaching/Jacobi_matrices.pdf

[19] Y. Yazlik and N. Taskara, A note on generalized k-Horadam sequence. Comput. Math. Appl. 63 (2012), pp. $36-41$. 